\title{
Magnetic fields in supernova remnants and pulsar-wind nebulae
}

\author{
Stephen P. Reynolds • B. M. Gaensler • Fabrizio Bocchino
}

Received: date / Accepted: date

\begin{abstract}
We review the observations of supernova remnants (SNRs) and pulsar-wind nebulae (PWNe) that give information on the strength and orientation of magnetic fields. Radio polarimetry gives the degree of order of magnetic fields, and the orientation of the ordered component. Many young shell supernova remnants show evidence for synchrotron X-ray emission. The spatial analysis of this emission suggests that magnetic fields are amplified by one to two orders of magnitude in strong shocks. Detection of several remnants in $\mathrm{TeV}$ gamma rays implies a lower limit on the magneticfield strength (or a measurement, if the emission process is inverse-Compton upscattering of cosmic microwave background photons). Upper limits to $\mathrm{GeV}$ emission similarly provide lower limits on magnetic-field strengths. In the historical shell remnants, lower limits on $B$ range from 25 to $1000 \mu \mathrm{G}$. Two remnants show variability of synchrotron X-ray emission with a timescale of years. If this timescale is the electron-acceleration or radiative loss timescale, magnetic fields of order $1 \mathrm{mG}$ are also implied. In pulsar-wind nebulae, equipartition ar-
\end{abstract}

S.P. Reynolds

Physics Department, North Carolina State University

Raleigh, NC, USA

Tel.: +01-919-515-7751

Fax: +01-919-515-6538

E-mail: reynolds@ncsu.edu

B.M. Gaensler

Sydney Institute for Astronomy

School of Physics, The University of Sydney

NSW 2006, Australia

E-mail: bryan.gaensler@sydney.edu.au

Fabrizio Bocchino

INAF-Osservatorio Astronomico di Palermo

Piazza del Parlamento 1

90134 Palermo, Italy

E-mail: bocchino@astropa.inaf.it guments and dynamical modeling can be used to infer magnetic-field strengths anywhere from $\sim 5 \mu \mathrm{G}$ to $1 \mathrm{mG}$. Polarized fractions are considerably higher than in SNRs, ranging to 50 or $60 \%$ in some cases; magnetic-field geometries often suggest a toroidal structure around the pulsar, but this is not universal. Viewingangle effects undoubtedly play a role. MHD models of radio emission in shell SNRs show that different orientations of upstream magnetic field, and different assumptions about electron acceleration, predict different radio morphology. In the remnant of SN 1006, such comparisons imply a magnetic-field orientation connecting the bright limbs, with a non-negligible gradient of its strength across the remnant.

\section{Shell supernova remnants: review}

\subsection{Introduction}

Supernova remnants and pulsar-wind nebulae are prominent Galactic synchrotron sources at radio and, often, $\mathrm{X}$-ray wavelengths. The spatial and spectral analysis of the synchrotron emission can be used to deduce or constrain magnetic-field strengths and orientations. In this review, "supernova remnant" (SNR) will be used for shell (i.e., non-pulsar-driven) remnants; if the emission is due to a pulsar, whether the object is young (like the Crab Nebula) or old (as in $\mathrm{H} \alpha$ bow-shock nebulae) the object will be termed a pulsar-wind nebula (PWN). Thus a SNR may contain a PWN, a combination sometimes called a "composite" SNR.

SNRs are primarily radio objects. In the Milky Way, 274 are listed in Green's (2009) well-known catalogue (http://www.mrao.cam.ac.uk/surveys/snrs/); most are known only by radio emission. SNRs are well-studied 
only in Local Group galaxies where they are bright and large enough to be well-imaged with radio interferometers. Galactic remnants range in angular size from less than $2^{\prime}$ to many degrees, and represent a range in ages from about 100 years $(\mathrm{G} 1.9+0.3)$ to over $10^{5} \mathrm{yr}$, though age estimates are difficult for very old objects.

\subsection{SNR Dynamics}

SNRs evolve through various phases as their shock waves decelerate in the ambient medium. Very soon after an explosion (either Type Ia or core-collapse) deposits $\sim$ $10^{51}$ erg into the ISM, a shock wave is driven into the surrounding circumstellar medium (CSM: modified by the progenitor) or unmodified interstellar medium (ISM). Core-collapse SNe eject several solar masses with a range of velocities, typically of order $5,000 \mathrm{~km} \mathrm{~s}^{-1}$. Type Ia's are thought to represent the thermonuclear disruption of a white dwarf, so eject $1.4 M_{\odot}$ at about $10,000 \mathrm{~km} \mathrm{~s}^{-1}$. Not long after the explosion, deceleration of the outer blast wave causes the formation of a reverse shock as inner ejecta are forced to decelerate. This begins the ejecta phase of evolution, characterized by the presence of both the forward shock (blast wave) heating ISM or CSM, and the reverse shock heating ejecta. Cooling timescales are much longer than dynamical timescales, so this phase is essentially adiabatic. Depending on the density structure in the ejecta and surrounding material, the shock radius may evolve as $t^{0.6}-t^{0.9}$ (Chevalier 1982). Eventually the reverse shock moves in to the center of the SNR and, after what may be extensive reverberations, disappears. By this time (several thousand years for typical parameters), several times the ejected mass have been swept up by the blast wave, and the remnant is settling into the Sedov phase, well described by the similarity solution for a point explosion in a uniform medium, with shock radius obeying $R \propto t^{0.4}$. This phase is adiabatic as well. Eventually, cooling times in shocked ISM become comparable to the remnant age, and shocks become radiative: lossy and optically prominent. This typically occurs when shock velocities $v_{s}$ drop to around $200 \mathrm{~km}$ $\mathrm{s}^{-1}$. Denser regions around the remnant periphery may become radiative before other parts; even young SNRs (like Kepler, SN 1604) can show radiative shocks in some regions. Once the bulk of the blast wave is radiative, the deceleration is more marked. The remnant interior will remain hot for some time, producing what is known as a "pressure-driven snowplow." Eventually, the remnant becomes sufficiently confused as to lose its identity, and the remaining kinetic energy is dissipated as sound waves in the ISM. Various blast-wave solutions are described in Ostriker \& McKee (1988); particular applications to SNRs are made in Truelove \& McKee (1999).

\subsection{Radio inferences}

The prevalence of radio emission from adiabatic-phase SNRs, with low shock compression ratios, indicates that particle acceleration must be ongoing; simple compression of ambient Galactic magnetic field and cosmic-ray electrons would produce both insufficiently bright emission and the wrong spectrum (Reynolds 2008). Since 1977, the traditional explanation for this electron acceleration has been diffusive shock acceleration (DSA; see Blandford \& Eichler 1987 for a comprehensive review). Electrons emitting synchrotron radiation at radio wavelengths have energies of order $1-10 \mathrm{GeV}$ $(E=14.7 \sqrt{\nu(\mathrm{GHz}) / B(\mu \mathrm{G})} \mathrm{GeV})$. The observed radio power-law spectra $S_{\nu} \propto \nu^{-\alpha}$, with $\alpha \sim 0.5$ (see Figure 1), imply from synchrotron theory a power-law energy distribution of electrons $K E^{-s}$ electrons $\mathrm{cm}^{-3}$ $\operatorname{erg}^{-1}$ with $s=2 \alpha+1 \sim 2$. Radio observations do not allow the separate deduction of energy in electrons and in magnetic field; the synchrotron emissivity of this power-law distribution of electrons is proportional to $K B^{(s+1) / 2}$, or roughly to the product of energy density in electrons and in magnetic field. If particle and magnetic-field energies were in equipartition (the minimumenergy state producing an observed spectrum), the magneticfield strength could be deduced. For a spherical remnant of radius $R$ pc at distance $D$ kpc with $\alpha=0.5$,

$B=20\left((1+k) S_{9} D_{\mathrm{kpc}}^{2} /\left(\phi R_{\mathrm{pc}}^{3}\right)\right)^{2 / 7} \mu \mathrm{G}$,

where $S_{9}$ is the flux density at $1 \mathrm{GHz}$ in $\mathrm{Jy}, \phi$ is the volume filling factor, and $k$ is the ratio of energy density in ions to that in electrons (e.g., Pacholczyk 1970). But there is no obvious reason that equipartition should hold: SNRs are typically very inefficient synchrotron radiators, with a very small fraction of the $10^{51}$ erg required to produce the observed emission. Thermal energies are very much larger than either particle or field energy. Thus equipartition magnetic-field strengths are really just a measure of (approximately) remnant surface brightness.

The question of magnetic-field amplification in strong SNR shocks, to be discussed at length below, is intimately related to the question of shock modification by cosmic-ray pressure (efficient shock acceleration, or nonlinear DSA). Thus evidence for nonlinear DSA is related to estimation of magnetic-field strengths. One prediction of this theory is the gradual deceleration of incoming flow (in the shock frame) by forward-diffusing cosmic rays, so that the compression ratio $r$ varies continuously from a possibly large value (far upstream ve- 
SNR Spectral Indices

(Green 2009)

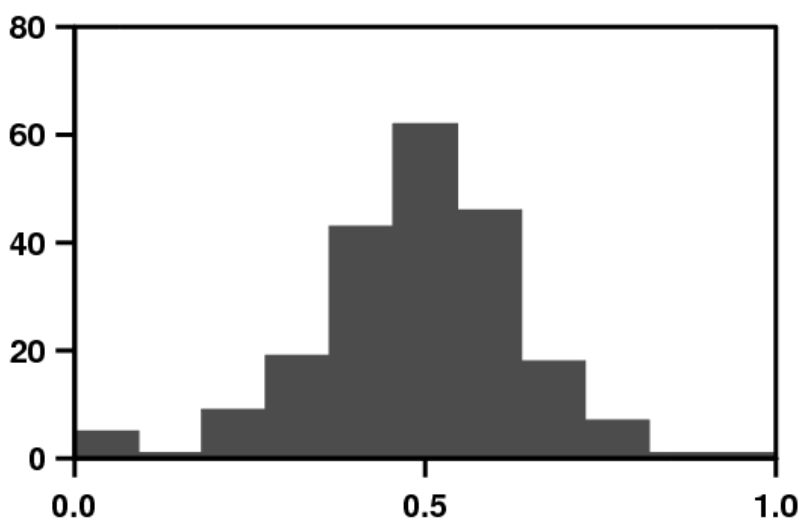

Fig. 1 Histogram of shell SNRs with fairly well-measured radio spectral indices, from Green 2009. PWNe are excluded.

locity/downstream velocity, perhaps 10 or more) to about 3 , at which point most calculations show the development of a "thermal subshock" on a length scale of thermal proton gyroradius, much smaller than other length scales. (See Figure 2.) In test-particle (inefficient) DSA, well-known results give the spectrum of accelerated particles as a power-law with energy index $s$ depending only on the shock compression ratio, $s=(r+2) /(r-1)$. Since strong adiabatic shocks are expected to have $r=$ 4 (for adiabatic index $\gamma=5 / 3$ ), we predict $s=2$ which implies $\alpha=0.5$, close to the mean of the distribution of over 200 SNR values from Green's catalogue. However, the dispersion in that distribution is substantial (see Figure 10; explaining values of $\alpha$ of $0.6-0.7$, typical for young SNRs, or as low as 0.3, sometimes seen in older SNRs, is not straightforward. Low Mach-number shocks will have lower compressions: in fact, for Mach number $\mathcal{M}, r^{-1}=(\gamma-1) /(\gamma+1)+2 /\left((\gamma+1) \mathcal{M}^{2}\right)$. For $\mathcal{M}<10$, this can significantly increase the steepness of the predicted spectrum; but this is demonstrably not the case in the young remnants with the steeper spectra. No variation in shock Mach number can explain $\alpha<0.5$; traditional explanations involve confusing thermal emission or other instrumental effects, but remain unconvincing.

A consistent explanation for steeper radio spectra in young remnants is available, however. Particles of a particular energy will diffuse (on average) a certain distance ahead of an efficient shock, where they will see a particular effective compression ratio, which fixes the slope of the distribution function near that energy. If, as expected, more energetic particles diffuse further, they will see larger compression ratios and the spectrum will become flatter. Thus one predicts spectra

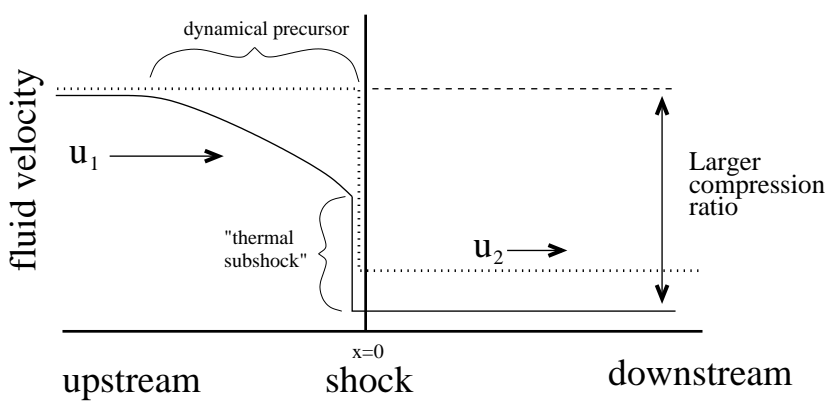

Fig. 2 Schematic velocity profile of a shock wave with upstream velocity $u_{1}$ and downstream $u_{2}$. Dotted line: test-particle shock (velocity discontinuity). Solid line: shock modified by cosmic rays.

flattening (hardening) to higher energy, an effect first pointed out by Eichler (1979). This effect was quantified in a Monte Carlo simulation of electron acceleration, and compared with radio observations of young SNRs, which did seem to show the effect (Ellison \& Reynolds 1991; Reynolds \& Ellison 1992; see Figure 3). The prediction for the emitted synchrotron spectrum depends on the magnetic-field strength; stronger fields mean lower-energy particles producing radiation in a particular radio-astronomical bandpass. Reynolds \& Ellison found that explaining the relatively steep spectra of Tycho's and Kepler's SNRs required magnetic fields of up to $1 \mathrm{mG}$ and above, though with substantial errors.

The degree of order in the magnetic field in SNRs can be directly inferred from the degree of polarization of synchrotron radiation (subject to observational issues such as Faraday depolarization and resolution effects [beam depolarization]). The degree of linear polarization of power-law synchrotron radiation from electrons in a uniform magnetic field is given by $P=(s+$ $1) /(s+7 / 3)$, so that $s=2 \Rightarrow P=69 \%$. Such high values are never observed in SNRs; a few older SNRs such as DA 530 show $P \sim 50 \%$ in some regions (Landecker et al. 1999, Figure 4), but historical shells show much lower values, 10 - 15\% (see references in Reynolds \& Gilmore 1993). Very roughly, the observed polarized fraction over the maximum possible gives an estimate of the fractional energy in the ordered component of the magnetic field Burn 1966); thus in Tycho, for instance (see Figure 5] [Reynoso et al. 1997]), less than $15 \%$ of the magnetic energy is in an ordered component. However, the direction of that component is radial, a peculiar property shared by the other historical shells SN 1006 , Cas A, Kepler, and G11.2-0.3. The nature of this radial component is unclear; while radial motions such as predicted to occur at the Rayleigh-Taylor unstable contact discontinuity between shocked ejecta and shocked ISM might enhance radial field, this effect should occur somewhat interior, while radial fields are observed im- 


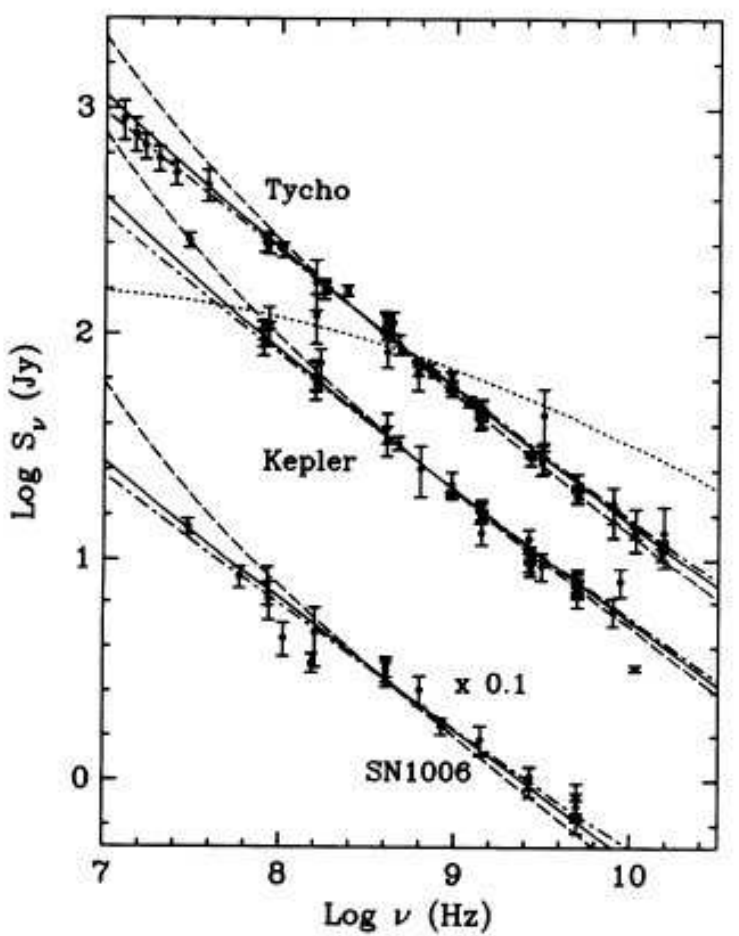

Fig. 3 Observed total flux observations of Tycho's and Kepler's SNRs and SN 1006, with model spectra superposed. All three spectra derive from the same electron spectrum but with varied magnetic field. For Tycho and Kepler, the dashed line corresponds to $B=10 \mathrm{mG}$, the solid line to $1 \mathrm{mG}$, and the dot-dashed line to $0.1 \mathrm{mG}$; for $\mathrm{SN} 1006$, the dashed, solid, and dot-dashed lines correspond to $B=10 \mathrm{mG}, 0.1 \mathrm{mG}$, and $1 \mu \mathrm{G}$, respectively. The dotted line shows the spectrum that would be produced if electrons had the same energy distribution as protons, showing how differently the nonlinear shock treats electrons and protons. (Reynolds \& Ellison 1992)

mediately at remnant edges (e.g., Tycho: Dickel et al. 1991). Various MHD simulations have been performed in an attempt to understand this effect (Jun \& Norman 1996; Jun \& Jones 1999). In older remnants such as DA 530 , magnetic fields are generally confused or tangential. Tangential fields can readily be explained by simple compression in radiative shocks with large $r$, and this is the conventional explanation.

\subsection{X-ray and gamma-ray inferences}

The discovery of X-ray synchrotron emission from SNRs dates from the early 1980's (Toor 1980; Becker et al. 1980; Reynolds \& Chevalier 1981) but observational and theoretical confusion prevented its wide acceptance until the unmistakable observations with ASCA of the remnant of SN 1006, demonstrating conclusively that central emission was thermal while the bright limbs showed lineless spectra well described by power laws (Koyama et al. 1996). (See Reynolds 2008 for a de-

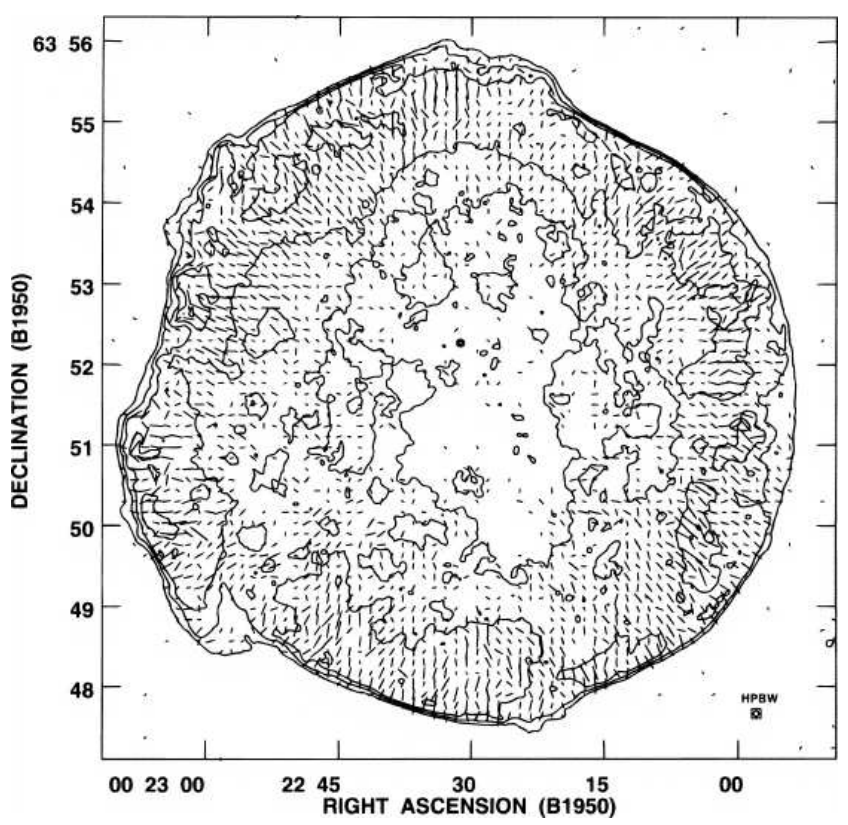

Fig. 4 Radio contours and polarized $E$-vectors at $1420 \mathrm{MHz}$ for DA 530 (Landecker et al. 1999). Direction of the sky-plane component of the magnetic field is at right angles to the directions of the $E$-vectors, i.e., predominantly tangential in the bright limbs. Foreground Faraday rotation has been corrected for, though it is small.

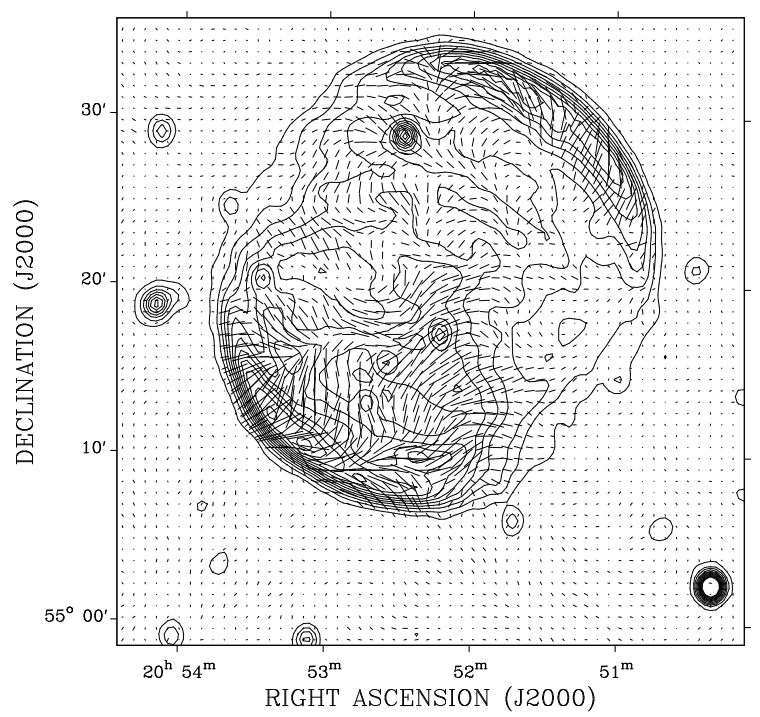

Fig. 5 Polarization vectors in Tycho's SNR at $1375 \mathrm{MHz}$ (Reynoso et al. 1997). These have been corrected for foreground Faraday rotation and rotated by $90^{\circ}$ to show the direction of the magnetic field.

tailed review of high-energy nonthermal emission from SNRs.) At this time, four Galactic SNRs show X-ray spectra dominated by synchrotron emission: G1.9+0.3, SN 1006, G347.3-0.5 (RX J1713.7-3946), and G266.21.2 ("Vela Jr." or RX J0852-4622). Of these, the first two are young, with symmetric X-ray morphologies, 
and show thermal X-ray emission from fainter regions. SN 1006 is widely accepted to be the remnant of a Type Ia supernova (evidence includes the presence of Balmerdominated optical emission from the forward shock, and the high Galactic latitude of $15^{\circ}$ ); the evidence is not as firm for G1.9+0.3, but it is also suspected to be a Type Ia remnant (Reynolds et al. 2008). The other two have much larger angular sizes and considerably more irregular morphologies (Slane et al. 1999, 2001); they also show no trace of thermal X-rays, putting severe upper limits on the presence of any thermal gas (Ellison et al. 2010). Both also contain X-ray point sources, suggesting a core-collapse origin. In addition to these four objects, all other historical shells (Tycho $=$ SN 1572, Kepler $=$ SN 1604, Cas A (unseen SN around 1680), G11.2$0.3=\mathrm{SN} 386$, and RCW $86=\mathrm{SN} 185 ?$ ) show synchrotron emission in regions, typically (but not always) "thin rims" at the remnant periphery. All, in addition to SN 1006, also show hard continua in the integrated X-ray flux seen by the (non-imaging) RXTE satellite (Allen. Gotthelf, \& Petre 1999). Detections above $8 \mathrm{keV}$ have also been reported by INTEGRAL (Cas A, Renaud et al. 2006; SN 1006, Kalemci et al. 2006; G347.30.5, Krivonos et al. 2007) and by non-imaging instruments BeppoSAX (Cas A to $80 \mathrm{keV}$; Favata et al. 1997) and Suzaku HXD (Cas A [to $40 \mathrm{keV}$ ], Maeda et al. 2009; G347.3-0.5 [to $40 \mathrm{keV}$ ], Tanaka et al. 2008; and Tycho [to $30 \mathrm{keV}$ ], Tamagawa et al. 2009). Arguments that the radiation is synchrotron essentially turn on the exclusion of all other possible processes (see arguments summarized in Reynolds 2008). Synchrotron photons at $4 \mathrm{keV}$ energy $\left(\nu \sim 10^{18} \mathrm{~Hz}\right)$ imply electron energies of order $100(B / 10 \mu \mathrm{G})^{1 / 2} \mathrm{TeV}$.

Power-law fits to the X-ray spectra are much steeper than radio spectra, indicating that the $\mathrm{X}$-rays come from the cutting-off tail of the electron distribution. Three limitations might be imagined for the maximum energy to which electrons can be accelerated due to DSA: finite age or size of the remnant, lack of MHD scattering waves above some $\lambda(\max )$, or radiative losses. The first two mechanisms would restrict ion acceleration as well, but radiative losses would affect electrons only. The cutoffs are likely to be exponentials or modified exponentials, so that the electron distribution is roughly given by $N(E)=K E^{-s} e^{-E / E_{\max }}$. In a uniform magnetic field, such a distribution would give rise to a spectrum $S_{\nu} \propto \nu^{-\alpha} e^{-\sqrt{\nu / \nu_{c}}}$ approximately, dropping off rather slowly. (A more careful calculation in the case of synchrotron losses predicts a spectrum with the same exponential factor $e^{-\sqrt{\nu / \nu_{c}}}$; Zirakashvili \& Aharonian 2007). Here $\nu_{c} \propto E_{\max }^{2} B$ is the "rolloff" frequency corresponding to the peak frequency emitted by electrons with energy $E_{\max }$.
In the cutoff part of the spectrum, we observe the competition between acceleration and loss rates. In standard DSA, the time for a particle to reach a relativistic energy $E$ (from $E_{i} \ll E$ ) is given by $\tau(E) \sim \kappa / u_{s}^{2}$ where $\kappa \equiv \lambda_{\mathrm{mfp}} c / 3$ is the diffusion coefficient. It is often assumed, but not demanded by the data, that $\kappa$ is "Bohm-like," that is, that the mean free path $\lambda_{\mathrm{mfp}}$ is proportional to the particle gyroradius $r_{g} \equiv E / e B$ (for extreme-relativistic energies). If we write $\lambda_{\mathrm{mfp}}=\eta r_{g}$, with $\eta$ the "gyrofactor," depending on the amplitude of scattering turbulence, then $\eta=1$ is Bohm diffusion and $\eta=$ const. $>1$ is "Bohm-like". In commonly used "quasilinear theory," $\eta(E) \equiv(\delta B / B)^{-2}$ where $\delta B$ is the amplitude of MHD turbulence resonant with particles of energy $E$. (So $\eta=$ const. means a particular spectrum of turbulence, equal energy per unit logarithmic bandwidth). Bohm diffusion is often taken as a limiting case, $\delta B \sim B$, but it has been argued that $\delta B \gg B$ is the possible outcome of some cosmic-ray driven instabilities (Bell \& Lucek 2001; Bell 2004). Relevant for using $\mathrm{X}$-ray spectra as a diagnostic of magnetic fields is that high fields give short mean free paths, small diffusion coefficients, rapid acceleration, and higher maximum particle energies.

Two classes of argument for high magnetic fields in young SNRs come from the last decade of observations with high-resolution, high throughput X-ray observatories: Chandra, XMM-Newton, and Suzaku. A morphological argument is based on the commonly seen phenomenon of "thin rims," in which synchrotron X-ray emission occurs at remnant peripheries in very narrow tangential features coincident with the shock location as inferred, e.g., from $\mathrm{H} \alpha$ observations (see Figure 6). Bamba et al. (2003) and Vink \& Laming (2003) argued that the small radial extent resulted from synchrotron losses on electrons, to infer strong amplification of $B$ :

$$
B \sim 200\left(u_{s} / 1000 \mathrm{~km} \mathrm{~s}^{-1}\right)^{2 / 3}(w / 0.01 \mathrm{pc})^{-2 / 3} \mathrm{G}
$$

(Parizot et al. 2006), where $w$ is the filament width. Values of $B$ from 60 to $230 \mu \mathrm{G}$ have been inferred for Tycho, Kepler, SN 1006, Cas A, and G347.3-0.5 (Parizot et al. 2006). These field strengths apply only to regions where X-ray synchrotron emission is seen, of course. An alternative explanation for the disappearance of synchrotron emission such a short distance behind the shock is that the magnetic field is primarily turbulent, and decays on a short lengthscale Pohl. Yan, \& Lazarian 2005). Tests attempting to discriminate between these two possibilities somewhat favor the radiative-loss explanation, but are not yet conclusive (Cassam-Chenaï et al. 2007). One particular difficulty is the presence of thin rims in radio images as well (see Figure 7); since radioemitting electrons have enormously longer loss timescales, 


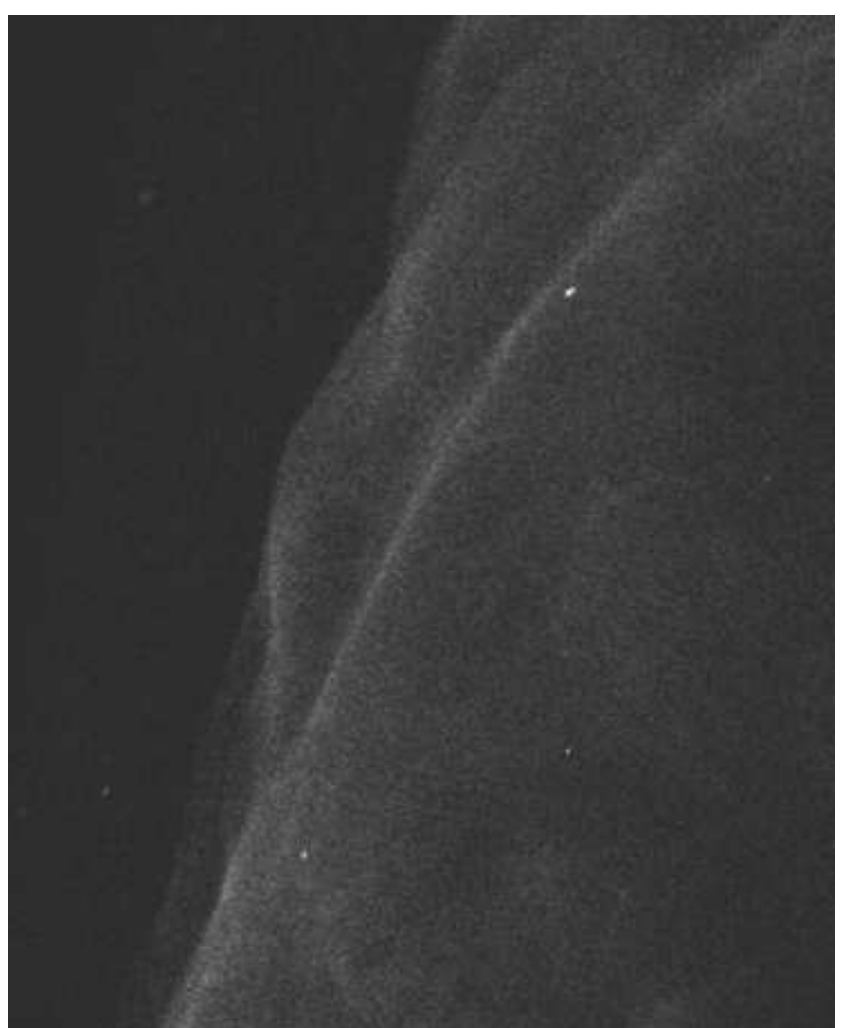

Fig. 6 Raw Chandra counts image of the NE region of the limb of SN 1006, showing "thin rims" of synchrotron radiation (Long et al. 2003). The brightness contrast of the very sharp rims is about a factor of 2 . The inner rim has a a clear $\mathrm{H} \alpha$ counterpart [Winkler et al. 2003].

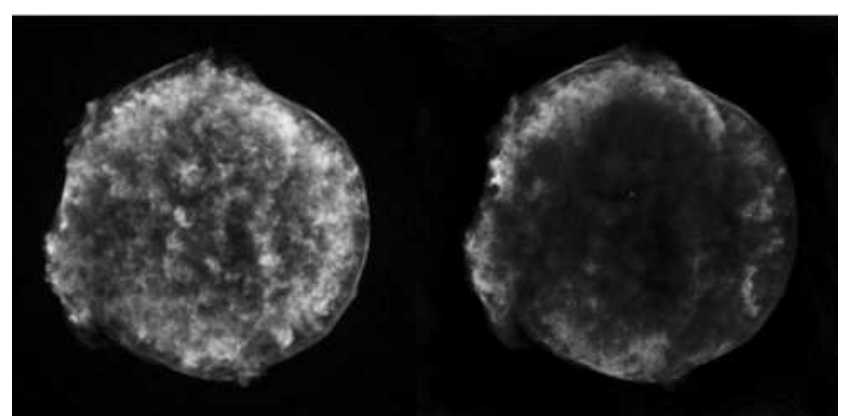

Fig. 7 Left: Chandra image of Tycho's SNR (CXC). Right: VLA image at $1420 \mathrm{MHz}$ (Reynoso et al. 1997). Note the thin rims in the X-ray image (e.g., NE quadrant), with some thin radio rims as well.

disappearance of the magnetic field is the only alternative. It is possible that both processes operate; theoretical models for strong magnetic-field amplification in modified shocks produce turbulent magnetic field which might well have some decay mechanism to compete with electron energy losses.

Another argument for (locally) strong magnetic fields is the discovery of variations in brightness on timescales of a few years of small features in X-ray synchrotron emission in Cas A (Patnaude \& Fesen 2007) and G347.3-
0.5 (Uchivama et al. 2007). Again, factors of 2 drop in brightness on a timescale of years require $B \sim 1 \mathrm{mG}$. Brightness increases on similar timescales are also observed; demanding acceleration times of years gives similar estimates for $B$. However, very strong levels of magnetic turbulence also naturally predict these kinds of fluctuations (Bykov, Uvarov, \& Ellison 2008; Bykov et al. 2009) without necessarily requiring quite as high values of $B_{\text {rms }}$. However, the remnant of SN 1006 shows no such small features or brightness variations (Katsuda et al. 2010), perhaps as a result of a Type Ia origin with more-or-less uniform surrounding material. (The absence of short-term variations does not imply absence of magnetic-field amplification; very high $B$ values could be generated in a quasi-steady state behind the shock, without producing short-term brightness fluctuations.)

New arguments relative to magnetic-field strengths in SNRs have resulted from the new generation of GeVTeV observational capabilities. The Fermi Gamma-Ray Space Telescope has been mapping the sky between about 0.2 and $300 \mathrm{GeV}$ since its launch in 2008, with gradually improving statistics on any given region of sky. One important early result is the detection of Cas A between 0.5 and $50 \mathrm{GeV}$ (Abdo et al. 2010a, see Figure 8). The emission may be either leptonic, that is, electron bremsstrahlung plus inverse-Compton upscattering of local photon fields, or hadronic, from the decay into photons of $\pi^{0}$ mesons produced in inelastic collisions between cosmic-ray protons and local thermal gas. The bremsstrahlung and hadronic contributions require knowledge of the gas density; the IC contribution requires knowledge of appropriate photon fields. The leptonic model shown in Figure 8 assumes a mean density of $26 \mathrm{~cm}^{-1}$, which requires a mean magnetic field (averaged over the entire emitting region of Cas A) of about $0.12 \mathrm{mG}$. If the actual emission is hadronic, the IC contribution must be lower (fewer relativistic electrons), demanding a larger magnetic field to produce the observed synchrotron fluxes at radio energies. (This argument was originally made based on early upper limits above $100 \mathrm{MeV}$ from COS-B and SAS-2; Cowsik and Sarkar [1980] derived a lower limit of about $1 \mathrm{mG}$.)

Similarly, any $\mathrm{TeV}$ detection or upper limit places lower limits on the mean magnetic field in regions containing relativistic electrons. So far, four SNRs have been imaged with the HESS air-Čerenkov telescope array in Namibia. These include three of the synchrotrondominated remnants: SN 1006, G347.3-0.5, and Vela Jr. (The fourth, G1.9+0.3, is too small for HESS imaging and too faint for a constraining upper limit). In addition, the (probably) historical shell SNR RCW 86 has been detected (Aharonian et al. 2009). In all cases, the $\mathrm{TeV}$ morphology tracks the X-ray morphology surpris- 


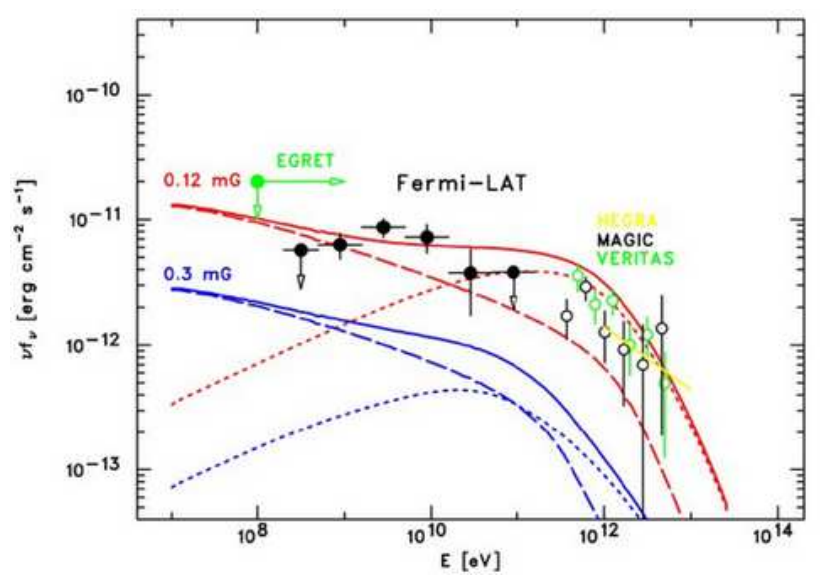

Fig. 8 Spectrum of GeV emission from Cas A as seen by Fermi (Abdo et al. 2010a). The solid curves are leptonic models (dots, IC; dashes, bremsstrahlung) with magnetic-field values shown. If the emission is actually hadronic, the magnetic-field strengths must be larger to suppress the IC component.

ingly closely. However, detailed attempts to model the emission as either due to hadronic or to leptonic processes run into significant problems. For hadronic models, required target gas densities seem to exceed measurements or upper limits on local thermal gas, while simple leptonic models do not do a good job reproducing the spectral shape and may also require implausibly small filling factors of magnetic field. However, lower limits to $\langle B\rangle$ do not depend much on the detailed modeling. In SN 1006 (Acero et al. 2010), $B \gtrsim 30 \mu \mathrm{G}$ is required; for G347.3-0.5, a leptonic model (Lazendić et al. 2004) demands a filling factor of magnetic field of only $1 \%$ to avoid overpredicting synchrotron emission. Within that $1 \%$ of the volume, the field is a modest $15 \mu \mathrm{G}$. (These estimates are relatively insensitive to model details; even a model that does not use DSA at all as a particle acceleration mechanism, but rather stochastic acceleration in the downstream region, fits the most recent $\mathrm{GeV}$ and $\mathrm{TeV}$ data for G347.3-0.5 with a leptonic model with $B \sim 12 \mu \mathrm{G}$; Fan, Liu, \& Fryer 2010). Hadronic models typically invoke much higher fields (Zirakashvili \& Aharonian [2010] find $B=127 \mu \mathrm{G}$ in G347.3-0.5).

HESS has also detected several other sources coincident with shell SNRs, such as CTB37B (HESS J1713381; Aharonian et al. 2008) and HESS J1731-347 (Acero et al. 2009). These may be further members of the nonthermal-X-ray class, older SNRs interacting with molecular clouds, or PWNe. They await further observational clarification.

The question of magnetic-field amplification is intimately connected with that of efficient particle acceleration through the proposals of Bell \& Lucek (2001) and Bell (2004) that cosmic-ray driven instabilities can

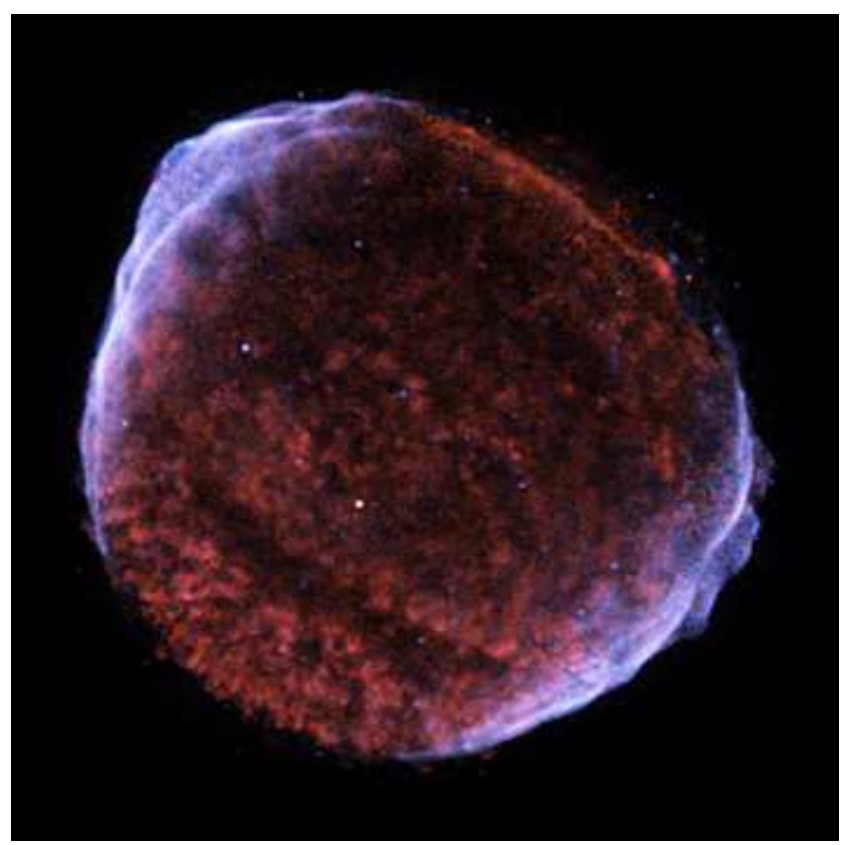

Fig. 9 SN 1006 with Chandra (NASA/CXC). Red, $0.50-0.91$ $\mathrm{keV}$; cyan, $0.91-1.34 \mathrm{keV}$; blue, $1.34-3.00 \mathrm{keV}$. Blue-white rims are line-free synchrotron emission; red interior is dominated by oxygen ejecta.

greatly increase the magnetic-field strength. Evidence for high magnetic fields is then taken as indirect evidence for an energetically significant component of cosmic rays (which must of necessity be ions) accelerated in the shock. This argument can be reversed: if evidence is found for efficient ion acceleration, then magnetic fields are likely to be amplified.

\subsection{Obliquity dependence}

The nature of magnetic-field amplification at strong shocks is a subject of intense theoretical activity. Observations can provide powerful constraints on some aspects of such amplification. In particular, two SNRs, SN 1006 and G1.9+0.3, show very simple bilaterally symmetric morphology which may contain clues to the dependence of particle acceleration and magnetic-field amplification processes on the shock obliquity angle $\theta_{\mathrm{Bn}}$ between the mean upstream magnetic field direction and the shock velocity. Section 3 discusses in detail a model for the radio morphology of SN 1006, as representative of this bilaterally symmetric class of SNRs (BSNRs).

The most natural explanation for the bilateral symmetry evident in Figures 9 and 11 is the variation in $\theta_{\mathrm{Bn}}$ one would encounter at different locations as a spherical shock encounters a roughly uniform magnetic field. If the field lies in the plane of the sky, the range in 


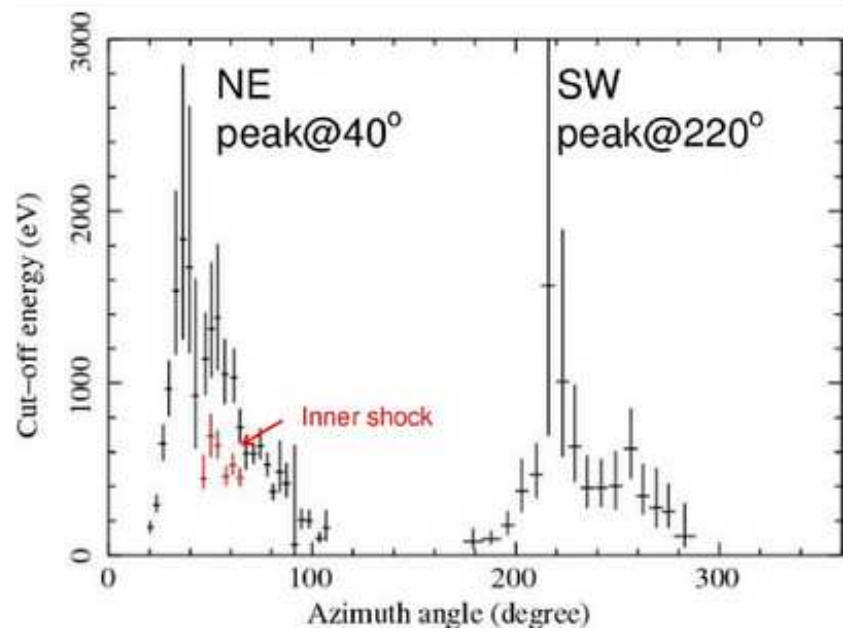

Fig. 10 Rolloff frequencies measured in 10" deep (radially; much larger in the azimuthal direction) rectangular regions around the outer edge of SN 1006 (red points: an interior rim) (Katsuda et al., in preparation). The exposure time is much longer in the $\mathrm{NE}$ region, so boxes are smaller.

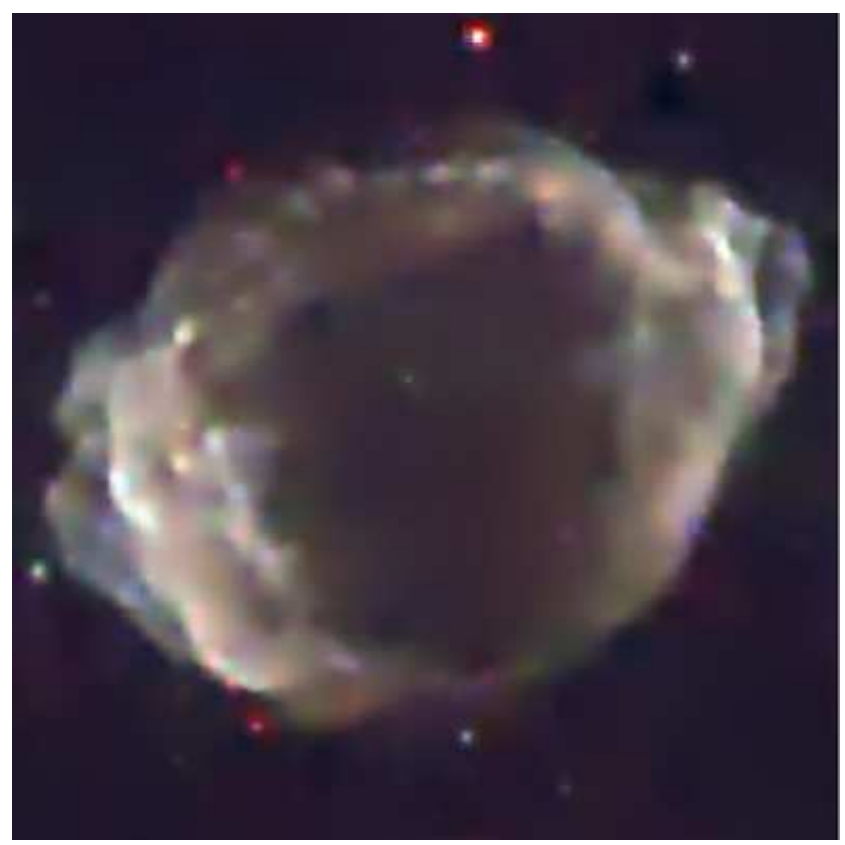

Fig. 11 G1.9+0.3, the Galaxy's youngest SNR (Chandra; Borkowski et al. 2010). Red, $1-3 \mathrm{keV}$; green, $3-5 \mathrm{keV}$; blue, $5-8 \mathrm{keV}$. Except for faint thermal emission in the center and north, the emission is dominantly synchrotron.

obliquities would be 0 to $\pi / 2$; if there is a line-of-sight component, the range drops until it is zero for $\mathbf{B}$ along the line of sight. Later in this chapter we describe a detailed calculation of the radio synchrotron morphologies resulting from different assumptions about the nature of the upstream magnetic field and of particle acceleration. Here we focus on the X-ray spectral variations each remnant displays.

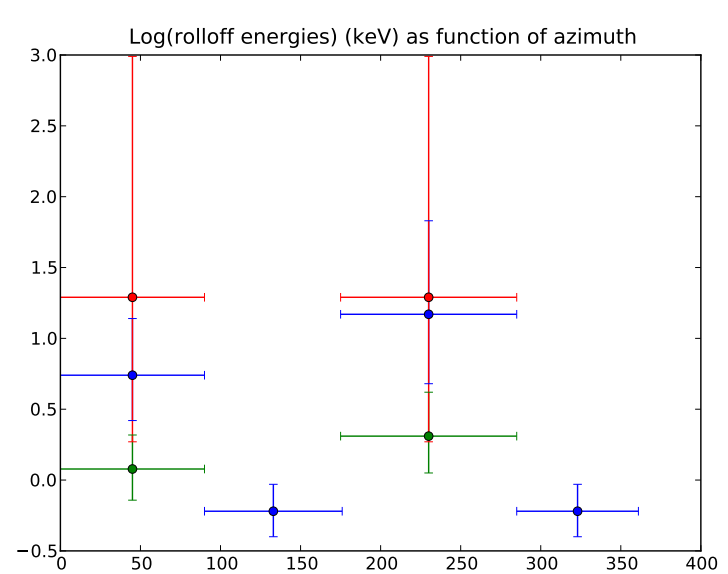

Fig. 12 Variations of $\nu_{\text {roll }}$ with radius and azimuth, for G1.9+0.3. Red: "Ears" extending beyond the main shell to E and W. Blue: main shell. Green: Just interior to bright regions of main shell. Note the similar trend at much poorer resolution to that seen in Figure 10

A simple model adequately characterizing most synchrotron X-ray emission from shell SNRs is the "srcut" model in the XSPEC software package, which is the spectrum emitted by a power-law electron distribution with an exponential cutoff. The frequency corresponding to the cutoff energy $E_{\max }$ is $\nu_{\text {roll }}$, the "rolloff" frequency (so called because the synchrotron spectrum from this distribution actually steepens rather gradually). $\nu_{\text {roll }}$ serves as a good indicator of the maximum energy reached by electrons at different locations, although depending on the mechanism limiting that maximum energy, $\nu_{\text {roll }}$ has different dependencies on physical parameters.

If the acceleration rate of particles varies with shock obliquity (independently of any obliquity-variations of the magnetic field), we can parameterize such effects in terms of a quantity $R_{J}: R_{J}\left(\theta_{\mathrm{Bn}}, \eta, r\right) \equiv \tau\left(\theta_{\mathrm{Bn}}\right) / \tau\left(\theta_{\mathrm{Bn}}=\right.$ $0)$. (This means that the value of $\eta$ is that for $\theta_{\mathrm{Bn}}=$ 0 ; any obliquity-dependence is subsumed into $R_{J}$.) We scale to typical values for young SNRs: $u_{8.5} \equiv u_{\mathrm{sh}} / 3000$ $\mathrm{km} \mathrm{s}^{-1} ; t_{3} \equiv t / 1000 \mathrm{yr} ; B_{10} \equiv B / 10 \mu \mathrm{G}$; and $\lambda_{17} \equiv$ $\lambda_{\max } / 10^{17} \mathrm{~cm}$. Then the values of $\nu_{\text {roll }}$ for each limiting mechanism obey (Reynolds 2008)

$$
\begin{aligned}
h \nu_{\text {roll }}(\text { age }) & \sim 0.4 u_{8.5}^{4} t_{3}^{2} B_{10}^{3}\left(\eta R_{J}\right)^{-2} \mathrm{keV} \\
h \nu_{\text {roll }}(\text { esc }) & \sim 2 B_{10}^{3} \lambda_{17}^{2} \mathrm{keV} \\
h \nu_{\text {roll }}(\text { loss }) & \sim 2 u_{8.5}^{2}\left(\eta R_{J}\right)^{-1} \mathrm{keV} .
\end{aligned}
$$

The effective limiting mechanism is the one producing the lowest value of $E_{\max }$; this may vary around the remnant periphery. Note that if radiative losses limit electron acceleration, the resulting $\nu_{\text {roll }}$ is independent of the magnetic-field strength. 
Figure 10 shows the variation in $\nu_{\text {roll }}$ around the periphery of SN 1006 (Katsuda et al., in preparation); similar data for G1.9+0.3 (Fig. 11) at much lower resolution (Reynolds et al. 2009) are shown in Figure 12 Variations by large factors occur. Equations $3-5$ above show that achieving variation by over an order of magnitude is not easy, especially for loss-limited acceleration. Even in the absence of any cosmic-ray-driven magnetic-field amplification, one would expect a variation in $B$ by about the shock compression ratio (4 or more) simply due to compression of the tangential component of $B$ due to flux-freezing. While there are no comprehensive predictions, calculations of magneticfield amplification normally assume parallel shocks $\left(\theta_{\mathrm{Bn}} \sim\right.$ 0 ), with the process being less effective at higher obliquities. The strong $B$-dependence for age or escape-limited acceleration could easily explain the observations - but with the serious side effect of predicting that the resulting maximum energies $\left(E_{\max } \sim 10-100 \mathrm{TeV}\right)$ would apply to ions as well as electrons, putting at least these two SNRs out of the running for producing cosmic rays up to the "knee", the slight steepening in the integrated spectrum of Galactic cosmic rays at Earth. Loss-limited maximum energies do depend on the shock speed, but the required variation by over 30 in $\nu_{\text {roll }}$ would demand an implausible factor of 5 variation in the current shock velocity. Anisotropic diffusion (Jokipii 1987) could produce more rapid acceleration at quasi-perpendicular shock than quasi-parallel $\left(R_{J}\left(\theta_{\mathrm{Bn}}\right)<R_{J}(0)\right)$, easily explaining the required amount of variation (Figure 13). However, most workers believe that this effect may not occur in highly turbulent media. In addition, electron injection into the DSA process may be more difficult at quasi-perpendicular shocks. As we show later in this chapter, the radio morphology may be more accurately reproduced with a quasi-parallel model, leaving the explanations of Figures 10 and 12 as an open question.

\subsection{Summary}

We summarize here the inferences on magnetic-field strength and geometry described above.

1. From radio observations, equipartition values of magnetic field strength are in the $\sim 10 \mu \mathrm{G}$ range, but there is little physical motivation to assume equipartition.

2. Radio polarization studies show that in young SNRs, the magnetic field is largely disordered, with a small radial preponderance. In older, larger SNRs, the field is often disordered but sometimes tangential.

3. Curvature (spectral hardening to higher frequency) is observed in the radio spectra of Tycho and Kepler.
Azimuthal variation of rolloff frequency

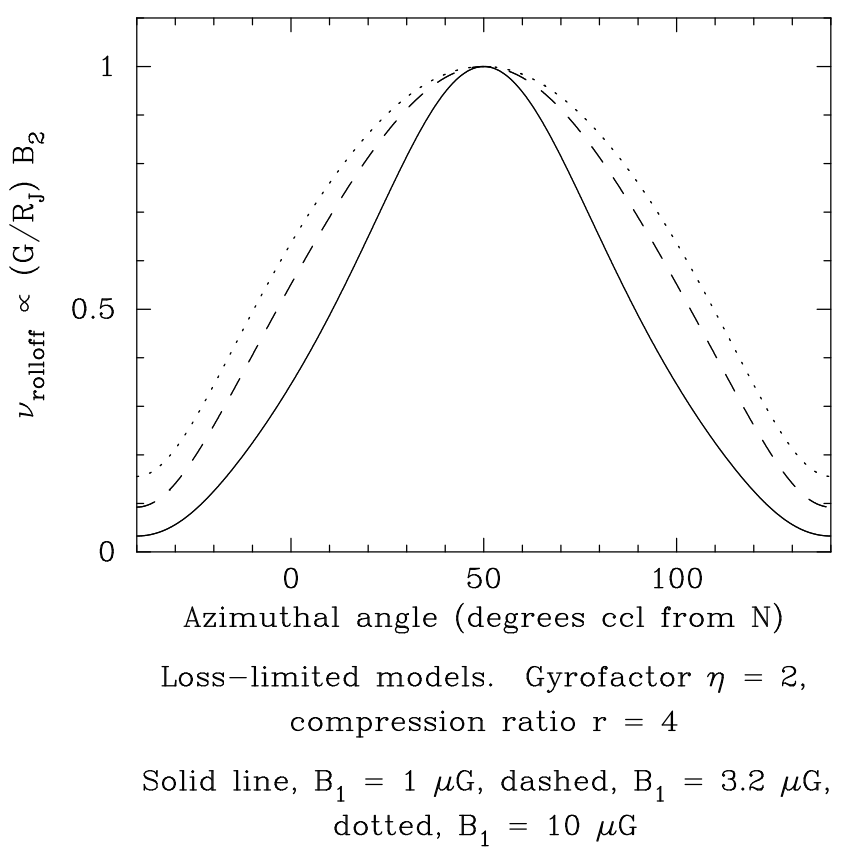

Fig. 13 Variation of $\nu_{\text {roll }}$ with azimuth (Revnolds 1998), according to the anisotropic diffusion model of Jokipii (1987). The required amount of variation of $\nu_{\text {roll }}$ can be produced, but only if the shock is perpendicular at the bright limbs. $B_{1}$ and $B_{2}$ are the upstream and downstream magnetic-field strengths, and $G$ is the ratio of an electron's energy-loss time in a field $B_{1}$ to that in the actual obliquity-dependent combination of $B_{1}$ and $B_{2}$ as it s scatters back and forth across the shock; see Reynolds 1998.

A nonlinear shock acceleration model can explain this with magnetic field strengths of $0.1-1 \mathrm{mG}$ (average over the emitting regions).

4. Thin rims of X-ray synchrotron emission in a few young remnants require $B \sim 50-200 \mu \mathrm{G}$ in the rims, if they are due to synchrotron losses on downstream-convecting electrons. However, thin radio rims are sometimes seen as well; they require that the magnetic field disappear somehow, presumably because it is a wave field which damps.

5. Brightening and fading of small X-ray synchrotron features in G347.3-0.5 and Cas A require $B \sim 1 \mathrm{mG}$, if they represent acceleration and loss times for electrons. Fields smaller by a factor of several are possible if the fluctuations are due to strong magnetic turbulence.

6. Large azimuthal variations in the rolloff frequency in SN 1006 and G1.9+0.3 are difficult to explain for a conventional picture of loss-limited acceleration in parallel shocks.

7. For Cas $\mathrm{A}$, the detection at $\mathrm{GeV}$ energies with Fermi requires $B \gtrsim 0.1 \mathrm{mG}$ to avoid overproducing the $\mathrm{GeV}$ emission with electron bremsstrahlung. 
8. TeV emission seen in four shell SNRs is not well explained by either leptonic or hadronic processes. However, if it is hadronic, the magnetic fields implied are of order $100 \mu \mathrm{G}$, while leptonic models require much lower fields.

\section{Magnetic fields in pulsar-wind nebulae}

\subsection{Introduction}

All young pulsars are slowing down in their rotation. The corresponding rate of change of rotational kinetic energy is enormous, typically in the range $\dot{E}=10^{32}-$ $10^{39} \mathrm{ergs} \mathrm{s}^{-1}$. In most cases only a negligible amount of energy is contained in the electromagnetic radiation corresponding to the neutron star's observed pulsations. Rather, the bulk of the rotational power is usually deposited into a relativistic magnetized particle wind that flows outward from the pulsar.

External pressure will cause this wind to abruptly decelerate at a termination shock. Beyond the termination shock, the pulsar wind thermalizes in pitch angle and radiates synchrotron emission, resulting in a pulsar wind nebula (PWN). The best known PWN is the Crab Nebula (Fig. 14), powered by the central young pulsar B0531+21. The Crab Nebula radiates synchrotron emission across the electromagnetic spectrum. In Xrays, its extent is small, reflecting the relatively short synchrotron lifetimes of electrons emitting at these energies. The optical synchrotron emission is larger, reflecting the longer lifetimes of the corresponding electron population. And finally, the radio emission shows the full extent of the source, with a radiative lifetime exceeding the age of the source $(\sim 1000$ years $)$.

\subsection{Observational Properties}

PWNe and SNRs have a variety of common properties: they both result from a supernova explosion, and they both are characterized by their polarized radio synchrotron emission. This has led to considerable confusion in the literature. For example, the Crab Nebula is often described as a "supernova remnant", despite the shell or blast-wave that would normally correspond to a SNR remaining as yet unidentified in this source (Frail et al. 1995; Seward et al. 2006; Tziamtzis et al. 2009). Some PWNe have for historical reasons been cataloged as "filled-center supernova remnants" Green 2009), while others, discovered more recently, have been excluded from such lists. As we describe below, a young SNR may contain a PWN, a combination often called a composite remnant (e.g., G21.5-0.9; Fig. 15).

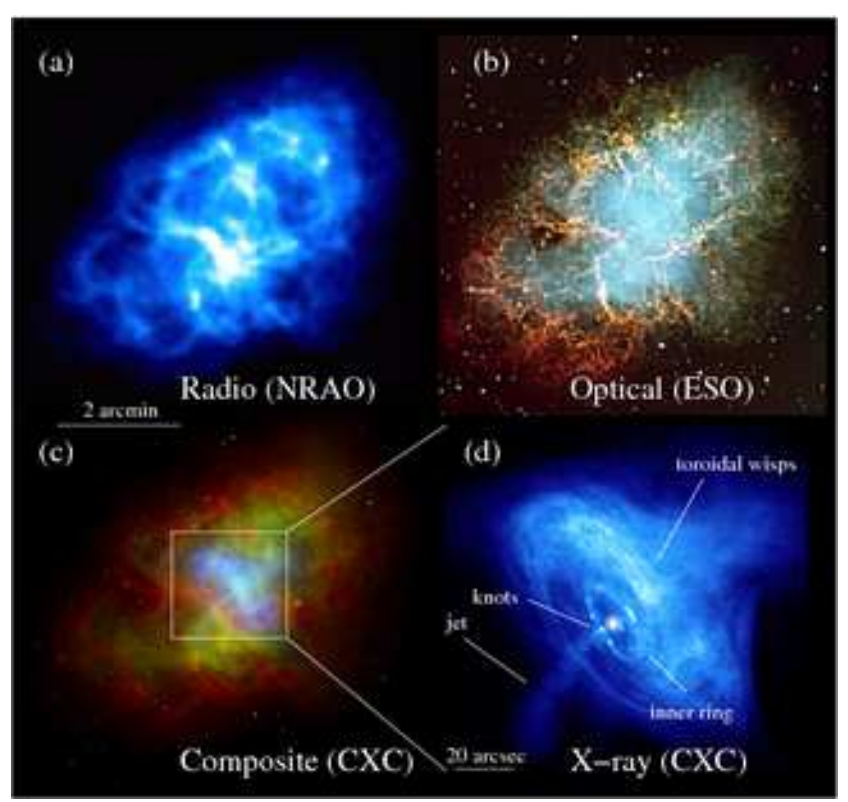

Fig. 14 Images of the Crab Nebula.

The key differences between SNRs and PWNe are as follows:

- Energy Source: SNRs result from an essentially instantaneous deposition of energy, in the form of a blast wave driven into the ISM by a supernova explosion. In contrast, PWNe have a continuous power source, the bulk relativistic flow of electron/positron pairs from an energetic neutron star.

- Radio Morphology: As a direct result of their differing sources of energy, SNRs and PWNe have distinct radio morphologies. SNRs are usually limbbrightened shells of synchrotron emission, while PWNe are typically amorphous or filled-center synchrotron nebulae, brightest at the pulsar's position.

- Radio Spectral Index: SNRs usually have relatively steep radio spectral indices, $\alpha \approx 0.3-0.8$, as shown in Figure 1. In contrast, PWNe have spectral indices in the range, $\alpha \approx 0-0.3$, which is too flat to be explained by simple models of diffusive shock acceleration (e.g., Atovan 1999; Fleishman \& Bietenholz 2007; Tanaka \& Takahara 2010).

- Angular Extent: SNRs are long-lived objects with a wide range of sizes, with angular extents ranging from $\sim 1^{\prime}$ to $>5^{\circ}$. PWNe are usually relatively small, with sizes in the range $10^{\prime \prime}$ to $30^{\prime}$, although a few older PWNe may be significantly larger (e.g., Vela X; Fig. 16).

- Fractional Polarization: At radio frequencies near $1 \mathrm{GHz}$, SNRs typically have modest amounts of linear polarization, at the level of 5\%-10\%. PWNe usually have very well-organized magnetic fields, with 


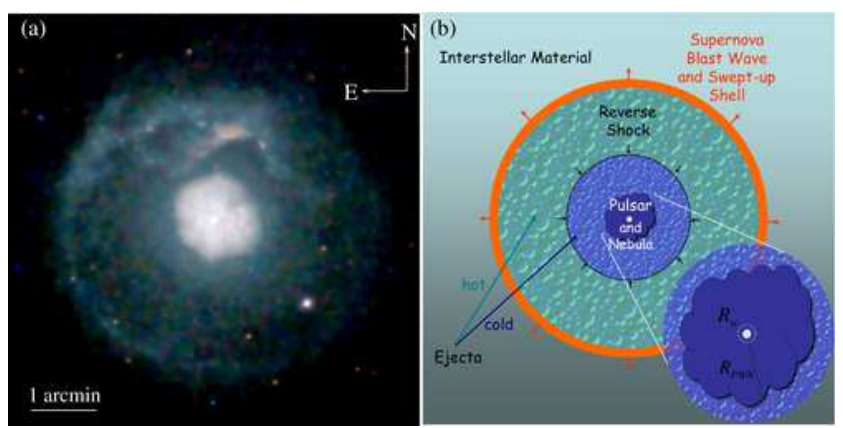

Fig. 15 Left: Deep Chandra image of the composite SNR G21.50.9 (Matheson \& Safi-Harb 2005). The PWN is the bright central condensation. Right: Schematic structure of a composite SNR containing a PWN

correspondingly higher polarization fractions, in the range $30 \%-50 \%$.

\subsection{PWN Evolution}

Theoretical expectations predict three main phases in the evolution of a PWN (Reynolds \& Chevalier 1984; Chevalier 1998, 2005), governed in turn by:

1. the expansion of the PWN;

2. interaction of the PWN with the surrounding SNR;

3. the motion of the pulsar powering the PWN.

Each of these phases is discussed in more detail below.

\subsubsection{Phase 1: Expansion into Unshocked Ejecta}

At early stages in a PWN's evolution, the pulsar's spindown luminosity remains relatively constant, so that there is a steady injection of energy into the PWN. As a consequence, the PWN expands supersonically into the surrounding low-pressure environment, with the radius, $R$, of the PWN evolving with time, $t$, as $R \propto t^{6 / 5}$ (Chevalier 1977). Thus the PWN drives a shock into the inner edge of the expanding SN ejecta (see Fig. 15).

Many pulsars are born with high space velocities, up to and beyond $1000 \mathrm{~km} \mathrm{~s}^{-1}$. However, because the sound speed inside the PWN is much higher than this, the PWN stays relatively centered on the pulsar, and has a quasi-spherical appearance.

Inside the PWN, there are two broad zones. Close to the pulsar is the unshocked wind zone, in which particles and Poynting flux flow freely outward from the central star. The pressure in this wind drops with increasing distance from the pulsar, until we reach a termination shock at which the wind is confined by external pressure. At this shock the wind particles are accelerated up to even higher Lorentz factors, and have their pitch angles scattered so that they can emit synchrotron radiation. Observations of this emission allow us to infer the composition of the wind at and immediately downstream of the shock. Specifically, the parameter $\sigma$ corresponds to the ratio of electromagnetic energy to particle energy at the wind shock, and is usually much less than one (Kennel \& Coroniti 1984; Gaensler et al. 2002): the shocked wind is particle-dominated $(\sigma \ll 1)$, in contrast to the unshocked wind which is expected to be radiation-dominated $(\sigma \gg 1)$.

Beyond the termination shock is the second main zone, the emitting region of the PWN, in which the bulk flow continues to decelerate to match the external boundary conditions, and where synchrotron emission is produced.

The morphology of a PWN in this early phase of evolution has been spectacularly revealed by X-ray images of the Crab Nebula taken by the Chandra X-ray Observatory (Weisskopf et al. 2000; Hester et al.|2002). These observations show that the Crab Nebula is dominated by a bright X-ray torus. The inner part of this torus is bounded by a bright X-ray ring, thought to correspond to the wind termination shock. To the south of the pulsar, an X-ray jet runs along the axis of the torus, apparently originating very close to the central pulsar before curving slightly at large distances from the center. A faint counter-jet can also be seen to the north.

The torus is thought to result from the Poynting flux concentrated in equatorial regions of the system by the wound-up magnetic field. However, a key point, first noted by Lyubarsky (2002), is that the termination shock radius should decrease with increasing angle from the equator. The axial jet seen for the Crab Nebula is therefore not a jet originating from the pulsar itself, but is part of the post-shock wind (e.g., Bogovalov \& Khangoulvan 2002).

Relativistic magnetohydrodynamic simulations show that at mid-latitudes, the external pressure can reverse the post-shock particle flow. The wind flows back along the surface of the termination shock toward the poles (Komissarov \& Lyubarsky 2003; Del Zanna et al. 2004, 2006), at which points it is collimated into jets by hoop stress (the "toothpaste effect; Camus et al. 2009). Simulations thus can do a reasonable job of reproducing the morphological properties of the Crab Nebula, as a result of a latitude-dependent termination-shock radius with a flow reversal.

Recently published optical polarimetry of the Crab Nebula using the Hubble Space Telescope has revealed spectacular details of this PWN's large-scale magnetic field (Hester 2008). A great deal of fibrous structure seen in polarization corresponds to the local field di- 


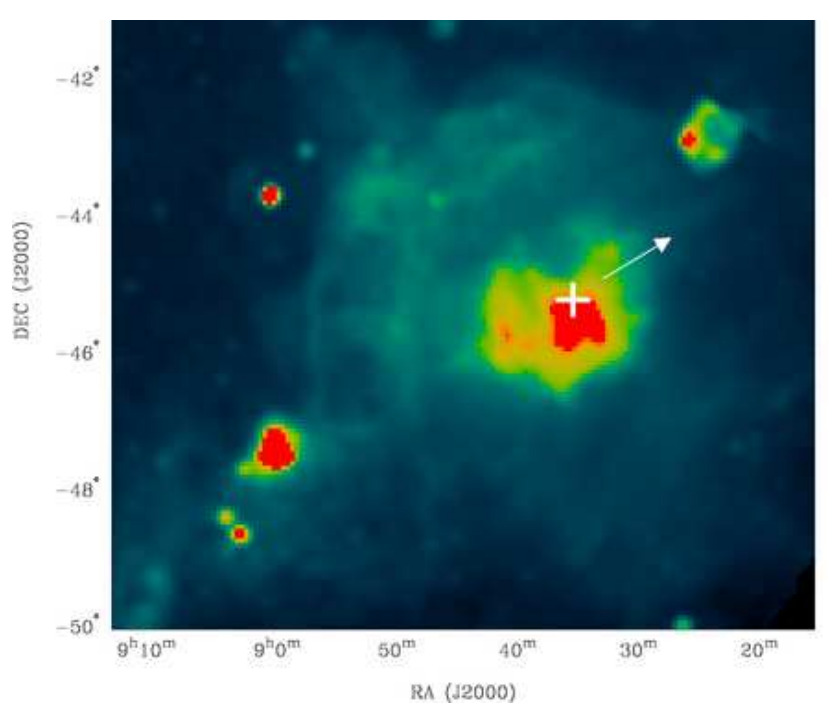

Fig. $162.4 \mathrm{GHz}$ Parkes image of the Vela supernova remnant (Duncan et al. 1996). The white cross indicates the pulsar, and the arrow its proper motion (Dodson et al. 2003); the fact that the pulsar is neither at nor moving away from the PWN's center indicates that the reverse-shock interaction has take place.

rection, while the torus of the nebula appears to be confined by a poloidal component of the field.

\subsubsection{Phase 2: Interaction with Reverse Shock}

A PWN moves into a new phase of evolution when its outer boundary collides with the reverse shock from the SNR in which it is embedded. This typically occurs after $\sim 7000$ years for the typical case of a $10^{51} \mathrm{erg}$ supernova ejecting 10 solar masses into an ambient ISM of density 1 atom $\mathrm{cm}^{-3}$ (Reynolds \& Chevalier 1984). The collision with the reverse shock initially compresses the PWN, and the system may reverberate for a couple of cycles before establishing a new equilibrium (Blondin et al. 2001; van der Swaluw et al. 2001; Gelfand et al. 2009)

The compression of the PWN raises the internal magnetic field, resulting in substantial synchrotron burnoff at high energies. The X-ray extent of a PWN in this phase can therefore be rather small. Furthermore, if the pulsar has a high space velocity or if the surrounding ISM is inhomogeneous, then the reverse shock does not collide with all parts of the PWN at the same time. As a result of this asymmetric collision, the pulsar can end up substantially offset from the center of its PWN, and the PWN's radio morphology can take on a chaotic or filamentary appearance (Blondin et al. 2001; van der Swaluw et al. 2004) (see Fig. 16).

\subsubsection{Phase 3: Supersonic Motion}

Since a pulsar moves ballistically while the surrounding SNR decelerates in its expansion, any pulsar with a

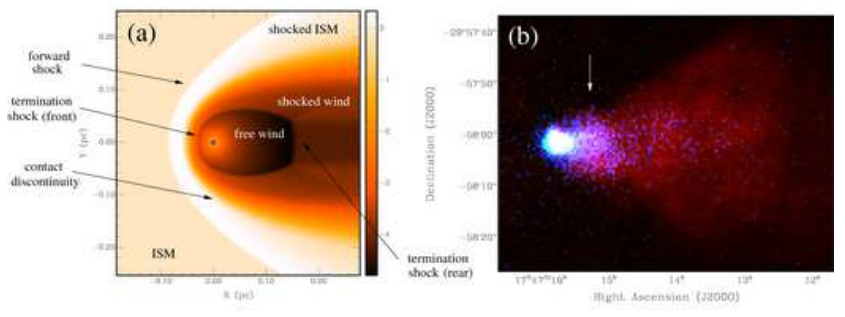

Fig. 17 Left: Hydrodynamic simulation of a pulsar bow shock. The pulsar (asterisk) is moving from right to left with Mach number 60. Right: Chandra image (blue) and VLA image (red) of G359.23-0.82 (the "Mouse"), the bow shock associated with PSR J1747-2958 Gaensler et al. 2004). The white arrow indicates a structure which may be the termination shock.

significant space velocity will eventually begin to move toward the SNR's rim. For a SNR in the Sedov phase, the sound-speed in the remnant's interior drops with increasing distance from the center. Specifically, van der Swaluw et al. (1998) has shown that for a Sedov SNR, the pulsar's motion will become supersonic when it has moved $68 \%$ of the way to the SNR's rim, which occurs at $50 \%$ of the total time taken for the pulsar to escape the SNR completely. Once the pulsar becomes supersonic, a new, bow-shock, PWN is formed, which no longer expands and which is externally confined by the ram pressure resulting from the pulsar's motion. Eventually the pulsar escapes the SNR completely, and now drives a bow shock through the ambient ISM (Bucciantini 2002; Chatteriee \& Cordes 2002; Gaensler et al. 2004; Bucciantini et al. 2005) (see Fig. 17).

\subsubsection{Measuring PWN magnetic fields}

We can infer the strength of the magnetic field inside PWNe through various techniques: by assuming equipartition between particles and magnetic fields averaged over an assumed synchrotron emitting volume (e.g., Hester et al. 1995), by measuring the radius of the termination shock from a high-resolution X-ray image and combining this with a physical model for the flow to estimate the field value at this location (e.g., Gaensler et al. 2002), or through observations of inverse Compton emission in $\mathrm{GeV}$ or $\mathrm{TeV}$ gamma-rays (e.g., MSH 15-52: Abdo et al. 2010b, Aharonian et al. 2005). De Jager \& Djannati-Ataï (2008) review GeV/TeV emission from PWNe. These approaches show that PWNe can have a wide range of nebular magnetic fields, from $\sim 5 \mu \mathrm{G}$ to $>1 \mathrm{mG}$. The magnetic field strength generally depends strongly on $\dot{E}, t$ and $\sigma$, as well as on the pulsar braking index. 
A variety of magnetic field orientations have been inferred in PWNe from the angles of their radio polarization. Some PWNe show a broadly toroidal magnetic field, some show a radial magnetic field, and in others there is a complex or tangled appearance. Kothes et al. (2006) have suggested that all PWNe have predominantly toroidal magnetic fields, but that these different polarization patterns result from differences in viewing angle. Toroidal polarization vectors, such as seen for the PWN G106.6+2.9, result when the observer looks along the pulsar's spin axis (Kothes et al. 2006). Radial fields, such as seen in the PWN G21.5-0.9 (Reich 2002) correspond to a viewing angle perpendicular to the spin axis. And more complex magnetic field morphologies, as for the PWN 3C 58 (Wilson \& Weiler 1976), occur for oblique viewing angles. The complex polarization properties of systems like 3C 58 may also occur as a result of kink instabilities, which can produce sheared loops of magnetic field throughout the nebula (Begelman 1998; Slane et al. 2004).

There are only limited observations of magnetic fields in pulsar bow shocks. The theoretical expectation is that the system forms a toroidal field near the apex of the bow shock, but that the magnetic field is collimated in the trailing magnetotail. Radio polarimetry matching this prediction has recently been observed by $\mathrm{Ng}$ et al. (2010) for the bow-shock PWN powered by PSR J15095850 . However, other magnetic field geometries have also been seen (e.g., Yusef-Zadeh \& Gaensler 2005), suggesting that the magnetic field structure of bow-shock PWNe may depend on whether the pulsar's spin axis aligns or mis-aligns with its velocity vector (e.g., Vigelius et a 2007).

\subsection{Outstanding Issues and Future Work}

There are many aspects of PWNe which are still active areas of investigation. Two particular areas of focus are as follows.

First, several PWNe have now been observed to show substantial time variability in their morphology and intensity (e.g., Pavlov et al. 2001; Hester et al. 2002; DeLaney et al. 2006; De Luca et al. 2007). These observations have provided a rich suite of information, but we now need relativistic magnetohydrodynamic simulations to fully understand the flow conditions and instabilities that produce these effects (Komissarov \& Lvubarskv 2004; Del Zanna et al. 2004; Bucciantini et al. 2005; Buccia 2010).

Second, while we have a multitude of observations of pulsar winds after they have been shocked, we have very limited data on the composition and transportation mechanism in the unshocked wind zone. Targeted observations and modeling of unique systems such as PSR J0737-3039A/B (the "double pulsar") may provide vital insights in this regard (McLaughlin et al. 2004; Lyutikov \& Thompson 2005).

\section{Constraints on the local interstellar magnetic field from radio emission of SN 1006}

\subsection{Introduction}

The radio morphology of supernova remnants (SNRs) may be very informative of the conditions of the magnetized environments in which the blast-wave expansion occurs and, in particular, on the acceleration processes at the shock front which give rise to the energetic electrons ultimately responsible of the synchrotron emission in the radio and (possibly) X-ray band. In this context, the bilaterally symmetric or barrel-shaped BSNRs Kesteven \& Caswell (1987), Gaensler (1998)) are considered ideal laboratories, because their morphology is definitely the result of the lack of small scale inhomogeneties which may confuse the interpretations. A point-like explosion in a uniform magnetized medium with constant strength and direction of $\mathbf{B}$ should give rise to a symmetric BSNR whose bright limbs are located where the magnetic field is parallel or perpendicular to the shock speed, if the injection efficiency is greatest where the shock is quasi-parallel or quasiperpendicular/isotropic, respectively, and if $\mathbf{B}$ lies in the plane of the sky, whereas different configurations al.eur at different aspect angles (Fulbright \& Revnolds $(1990))$.

However, in real life, BSNRs are often asymmetric, and therefore Orlando et al. (2007) (hereafter Paper I) have recently generalized the study of Fulbright \& Reynolds (1990) to the cases in which the explosions occur in a large scale gradient of density or magnetic field, showing that this model is able to reproduce most of the asymmetries observed in real BSNRs. In particular, the radio morphology loses one axis of symmetry, and the limbs are not equally bright (if the gradient runs across the limbs) or they are not opposite and they converge on the side in which the density or the magnetic field is increasing (if the gradient runs between and parallel to the limbs).

It is clear that morphology of BSNRs is tightly couandiedil to the magnetized environments in which the shock expands, and it is of particular interest to note here the preference of BSNR symmetry axes to be oriented parallel to the Galactic plane, as reported by Gaensler (1998). It seems therefore possible to study their morphology to derive the geometry of the surrounding mag- 
netic field, thus shedding more light on the microphysics of the particle acceleration processes at the shock front.

The remnant of SN 1006 seems to be the object in which this kind of study may be most fruitful. The uniform environment and the bright limbs visible in most of the electromagnetic spectrum make it a real case study in the field of particle acceleration mechanisms in strong shocks. Indeed, Rothenflug et al. (2004), using a simple and powerful geometrical argument applied to the XMM-Newton X-ray image of SN 1006, based on the ratio between the central and the rim luminosity, showed that if the remnant is cylindrically symmetric, the bright limbs are likely to be polar caps (instead of an equatorial belt) and that, therefore, the magnetic field is oriented perpendicular to the bright limbs, in the NE-SW direction. This argument seems to break the dichotomy between the two competing scenarios of the dependence of the electron injection efficiency or electron acceleration rate on $\theta_{\mathrm{Bn}}$, preferring a situation in which the injection is most efficient or acceleration most rapid when the field is along the shock speed (quasi-parallel scenario) over the situation in which the field is perpendicular (quasi-perpendicular scenario, see Fulbright \& Reynolds (1990) 1 .

On one hand, these findings were somehow expected since Völk et al. (2003) already pointed out that in SN 1006 the injection should be maximum at parallel shocks. However, in the light of the uncertainties related to the details of the acceleration processes, several authors still considered the quasi-perpendicular scenario a viable option: Fulbright \& Reynolds (1990) argued against the quasi-parallel scenario pointing out that quasi-parallel models often give rise to unobserved morphologies in the radio band; Yamazaki et al. (2004) still considered both models to explain the observed width of selected filaments of SN 1006 observed by Bamba et al. (2003), whereas Amano \& Hoshino (2007) developed a quasi-perpendicular model which agrees very well with the same data. Moreover, the same simple geometrical argument of Rothenflug et al. (2004), if applied to the radio image, would be in agreement with the equatorial belt (cfr. $R_{\pi / 3}=0.7$ in Sect. 3.2 of Rothenflug et al. (2004)). This discrepancy has never been explained in the literature and remain one of the most intriguing

\footnotetext{
1 Since X-ray synchrotron brightness depends on both the efficiency of electron injection into the acceleration process, and on the rapidity of acceleration to high energies, studies of the X-ray morphology involve a combination of both possible effects, while radio studies are insensitive to acceleration-rate issues, because acceleration to the $\mathrm{GeV}$ energies required for radio emission is always extremely rapid compared to evolutionary timescales. Thus variations of radio morphology with obliquity point to electron injection physics alone.
}

Table 1 MHD models of SN 1006 used in this work

\begin{tabular}{lc}
\hline Name & $\nabla \mid \mathbf{B}^{a}$ \\
\hline UNIFORM_B & 0 \\
GRAD1 & 1.4 \\
GRAD2 & 1.5 \\
GRAD3 & 2.0 \\
GRAD4 & 4.7 \\
\hline
\end{tabular}

$a$ The relative variation of the modulus of the magnetic field over a scale of $10 \mathrm{pc}$

open issues in the comparison between models and observations.

Recently, Petruk et al. (2009) have further investigated this issue, showing that, in the framework of a simple model of SN 1006 in terms of a point-like explosion occurring in a uniform density and uniform magnetic field medium, there is no way to reconcile the quasi-parallel scenario and the SN 1006 morphology as observed in the radio band. This contradiction between the radio morphology (suggesting quasi-perpendicular injection efficiency scenario) and X-ray morphology (suggesting quasi-parallel scenario) has prompted us to investigate the effects of non-uniformity of the environment on the observed properties of this remnant, capitalizing on the work of Orlando et al. (2007) on asymmetric BSNRs.

In Sect. 3.2, we will briefly describe the MHD model we have used to reproduce the remnant of SN 1006, which include a small gradient of the magnetic field. In Sect. 3.3, we introduce the methodology for the comparison between modeled and observed radio images of SN 1006, while in Sect. 3.4 we will discuss the results of the comparison, showing that, not only can the model invoking a gradient of $|\mathbf{B}|$ reconcile, for the first time, the radio and $\mathrm{X}$-ray morphology of the remnant, but it also provides stringent constraints on the overall geometry of the field in the vicinity of the remnant.

\subsection{The model}

Since there is accumulated evidence that the density around SN 1006 is fairly constant, with the exception of the NW sector where an encounter with a dense cloud is occurring (Acero et al. (2007), Miceli et al. (2009)), we argue that a model with a $|\mathbf{B}|$ gradient is more appropriate to describe the asymmetries of the limbs of SN 1006. The remnant was modeled as a point-like explosion of 1.4 solar masses of ejecta having a kinetic energy of $E=1.3 \times 10^{51}$ erg occurring in a uniform density medium with $n_{0}=0.05 \mathrm{~cm}^{-3}$. The ISMF has a value of $30 \mu \mathrm{G}$ in the environment of the explosion site and it is assumed to have a gradient along the $\mathrm{Y}$ axis. 


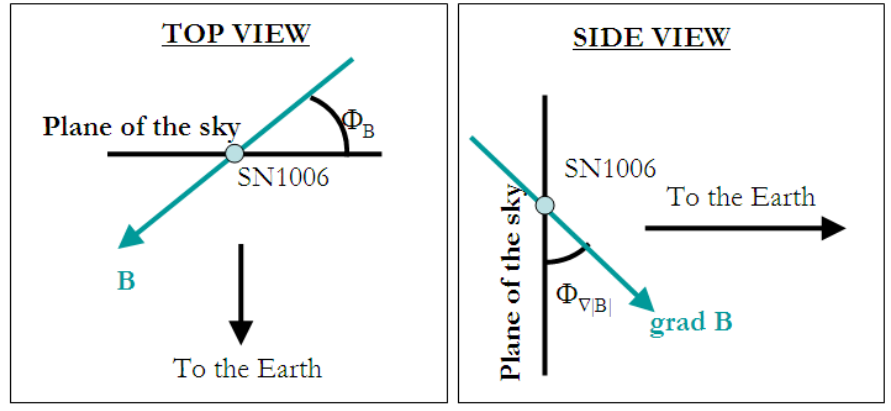

Fig. 18 Left panel: Definition of $\phi_{\mathbf{B}}$. Right panel: Definition of $\phi_{\nabla|\mathbf{B}|}$

Such a high value (for the location of SN1006 in the Galaxy) has been chosen in order to take into account as a first approximation the effects of magnetic-field amplification, to have a postshock $|\mathbf{B}|$ of the order of $\sim 100 \mu \mathbf{G}$ in our simulation, in agreement with observations of some SN1006 filaments. Moreover, the magnetic field has a gradient along the $\mathrm{Y}$ axis. The direction of the magnetic field is in the XY plane. $\nabla|\mathbf{B}|$ values used in our simulations are reported in Table1. The gradient in the initial conditions has been modeled with a dipole located along the $\mathrm{Y}$ axis, exactly as in Orlando et al. (2007), to which the reader is referred for further details on the model and numerical code used for the simulations. The simulations were stopped at $t=1002 \mathrm{yr}$, checking that the shock velocity and remnant radius are compatible with the observed values $\left(4600 \mathrm{~km} \mathrm{~s}^{-1}\right.$ and $9.6 \mathrm{pc}$, respectively).

\subsection{Comparison between models and observations}

\subsubsection{The angles defining the viewing geometry}

Several contributions in the literature show that the observed morphology of a SNR emitting synchrotron radiation is strongly affected by the angle between the dominant direction of $\mathbf{B}$ and the line of sight, $\phi_{\mathbf{B}}$. Moreover, in our circumstance, since we also have a gradient of the magnetic field, we are forced to consider the dependence of the observed morphology on the angle between the direction of the gradient and the plane of the sky, $\phi_{\nabla|\mathbf{B}|}$. The definitions of $\phi_{\mathbf{B}}$ and $\phi_{\nabla|\mathbf{B}|}$ are sketched in Figure 18. For the purposes of this work, we have chosen to synthesize our maps in the following way: first, we apply a rotation of $\phi_{\nabla|\mathbf{B}|}$ degrees around an axis passing through the center of the remnant and parallel to the limbs, where positive angles mean that regions of increasing $\mathbf{B}$ are closer to us. Next, we apply a rotation of $\phi_{\mathbf{B}}$ degrees around an axis passing

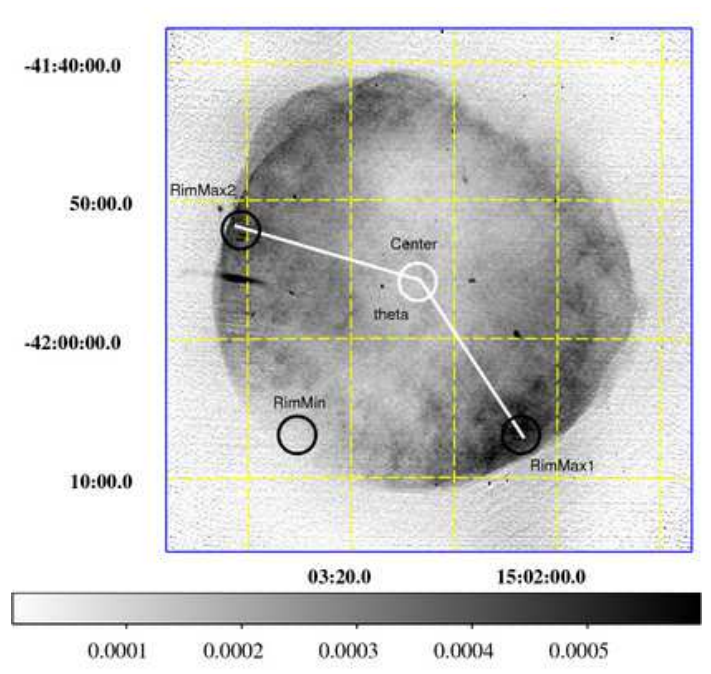

Fig. 19 Radio map of SN 1006 at $1.4 \mathrm{GHz}$, with the four regions in which the surface brightness of the remnant has been averaged for the purpose of computation of the four morphological parameters: $A=R I M_{\max } / R I M_{\min }, C=$ center $/ R I M_{\max }$, $R_{\max }=R I M_{\max 1} / R I M_{\max 2}$ and $\theta_{D}$, the aperture angle between the limb maxima. Adapted from Petruk et al. (2009).

through the remnant center and lying in the plane of the sky. Others rotation schemes give similar results. The adopted values of $\phi_{\mathbf{B}}$ are from $0^{\circ}$ to $90^{\circ}$ in steps of $2^{\circ}$ and the ones of $\phi_{\nabla|\mathbf{B}|}$ are from $0^{\circ}$ to $90^{\circ}$ in steps of $15^{\circ}$. Therefore, for each model, we have generated 315 maps encompassing all the combinations of the relevant angles.

\subsubsection{The morphological parameters}

The exploration of the parameter space is a challenging task, because it involves the morphological comparison between many model maps and the real SN 1006 images. We devised a simple procedure which involves the comparison of the value of 4 morphological parameters calculated both from the synthesized radio emission maps and from the observed radio image of SN 1006. The parameters are the ratio between the maximum and the minimum surface brightness $\left(S_{b}\right)$ around the $\operatorname{rim}(A)$, the ratio between the maximum around the rim and the center of the remnant $(C)$, the ratio of $S_{b}$ of the two bright limbs $\left(R_{\max }\right)$, and the angular separation between the limbs $\left(\theta_{D}\right)$. The parameters $A$, $R_{\max }$ and $\theta_{D}$ were also used in Orlando et al. (2007), and we refer to that paper for further discussion. The $C$ parameter is introduced here to measure the luminosity contrast between the brightest rim and the center. Note that the $C$ parameter does not correspond to the $R_{\pi / 3}$ parameter used by Rothenflug et al. (2004) to exclude the equatorial belt scenario for SN 1006, because $C$ is measured in a small circular region in the radio 
map (see below). The parameters $A, C$ and $R_{\max }$ are measured using the radio map of Petruk et al. (2009), by averaging the $S_{b}$ value in circular regions of $45^{\prime \prime}$ radius (Fig. 19). The values we obtained for the observed image of SN 1006 are $A=2.7 \pm 0.2, C=0.36 \pm 0.03$, $R_{\max }=1.2 \pm 0.1$, and $\theta_{D}=135^{\circ} \pm 10^{\circ}$. For the measurement of the parameters in the synthesized model radio maps, we used an automated procedure to find the maximum of the two limbs, the minimum between rims along the rim and the central position. Then we used an average in a circular region whose radius is the same percentage of the SNR radius as used in the real radio image $(5 \%)$. Since the model images have been synthesized using the same number of pixels per radius of the remnant, this procedure ensures that the model and observed values of the parameters are comparable.

\subsection{Results}

\subsubsection{Quasi-parallel scenario}

In Fig. 20, we show the $A-C, R_{\max }-C$ and $\theta_{D}-C$ scatter plots computed in the synthesized radio map of our SN 1006 model including a weak gradient of the magnetic field ( $\mathbf{B}$ varies by a factor of 1.4 over $10 \mathrm{pc}$ ), and considering a quasi-parallel scenario for the obliquity dependence of the electron injection efficiency. We also overplotted the values of the parameters derived using the real radio map of the remnant of Petruk et al. (2009). Though the agreement is not exactly perfect in all the plots, we can note that we can define a very limited region of the parameter space $\phi_{\mathbf{B}}-\phi_{\nabla|\mathbf{B}|}$ which is compatible with the observed values of $A, C, R_{\max }$ and $\theta_{D}$. This means that the observed radio morphology of SN 1006 is overall compatible with a quasi-parallel scenario for this remnant, if we include a weak gradient of $\mathbf{B}$. A nonzero gradient is an essential ingredient to reconcile the radio morphology with the quasi-parallel scenario, and this is shown in Fig. 21, in which we show the $A-C$ scatter plot for various $\phi_{\mathbf{B}}$ angles, in the uniform $\mathbf{B}$ case and quasiparallel, quasi-perpendicular and isotropic scenario 2 . In this case, this plot suggests that quasi-parallel models do not reproduce the observed parameters at all, unlike the quasi-perpendicular and isotropic scenarios, as already pointed out by Petruk et al. (2009).

Let us now come back to Fig. 20. A remarkable result is that the comparison between the quasi-parallel model and the observation strongly excludes a situation in which the polar caps are in the plane of the sky $\left(\phi_{\mathbf{B}}=90^{\circ}\right)$ or along the line of sight $\left(\phi_{\mathbf{B}}=0^{\circ}\right)$.

\footnotetext{
${ }^{2}$ In the uniform $\mathbf{B}$ case, $R_{\max }=1$ and $\theta_{D}=180^{\circ}$ always.
}
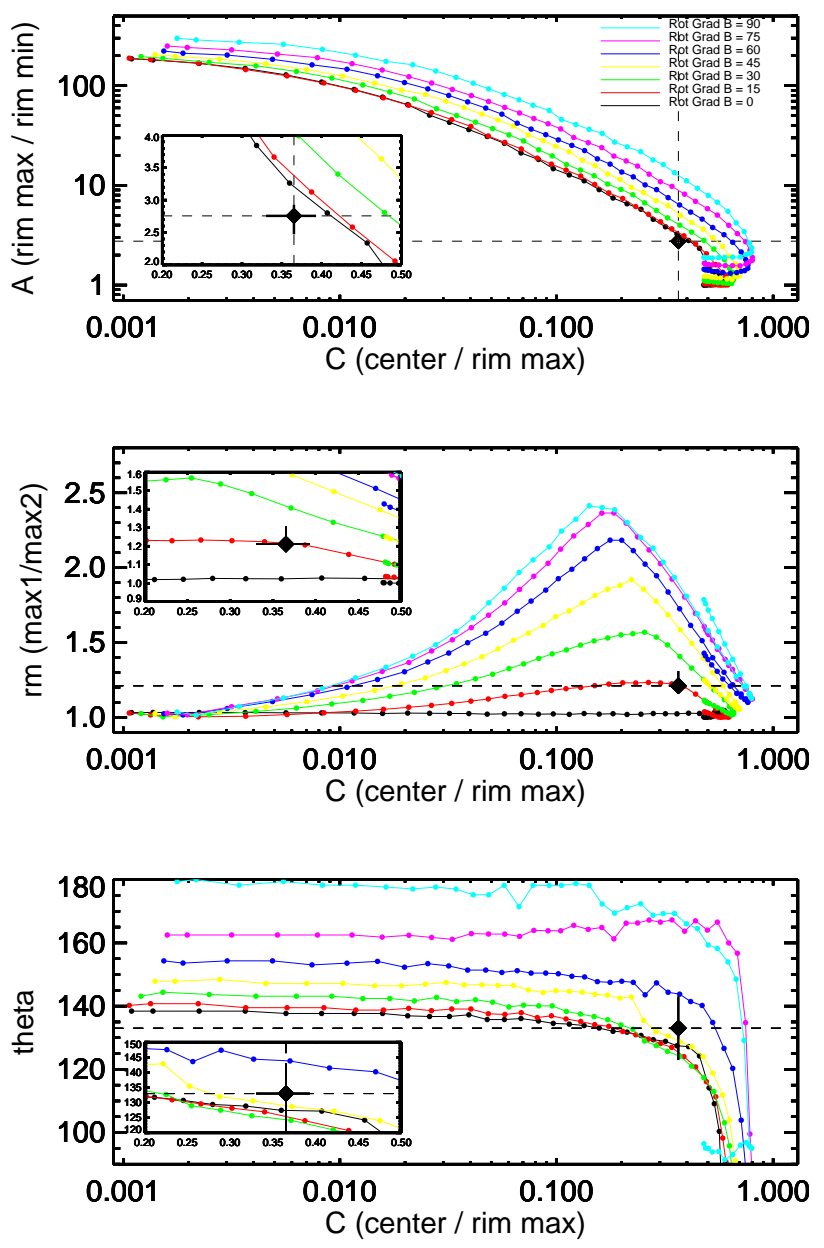

Fig. 20 Top panel: $A-C$ scatter plot for our models, assuming the quasi-parallel scenario and an age of $1000 \mathrm{yr}$. Different colors correspond to different values of $\phi_{\nabla|\mathbf{B}|}$, where each dot corresponds to a given value of $\phi_{\mathbf{B}}$ (from $0^{\circ}$ to $90^{\circ}$ in steps of $2^{\circ}$. We overplotted the $(A, C)$ values measured for SN 1006 (the inset shows a zoom near this point). Middle panel: Same as top panel but for the $R_{\max }-C$ pair. Lower panel: Same as top panel but for the $\theta_{D}-C$ pair.

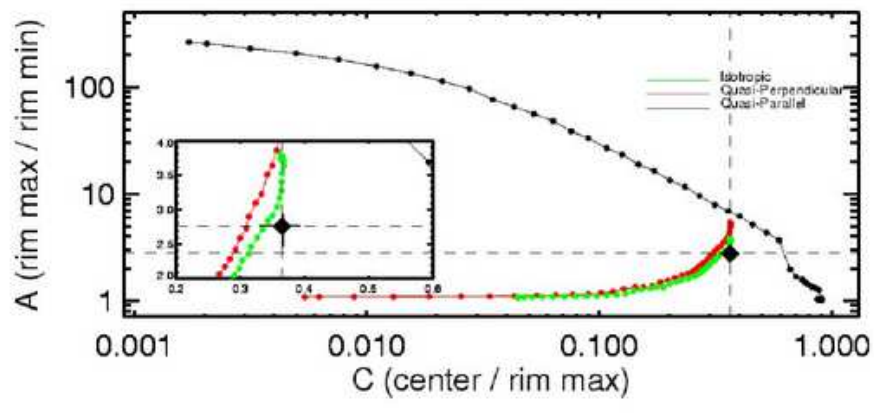

Fig. 21 Same as Fig. 20 top panel, but for the model with uniform magnetic field. 

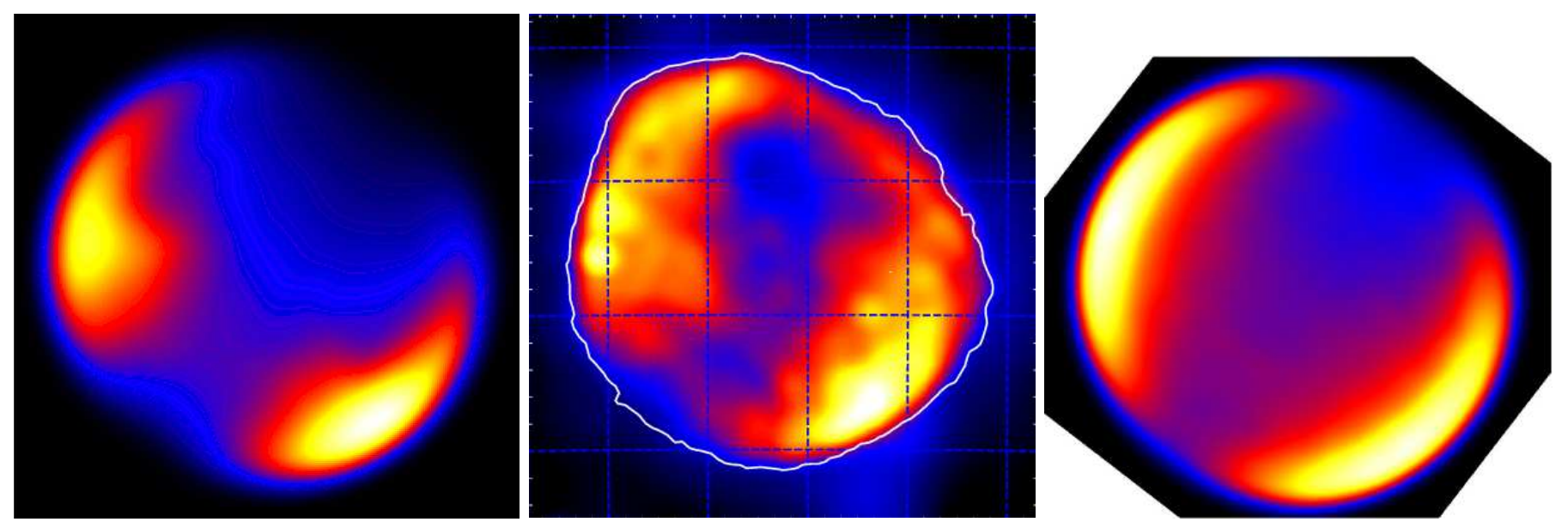

Fig. 22 Left panel: Synthesized radio image at $1 \mathrm{GHz}$ for our best-fit model (GRAD1 in Table 1 and quasi-parallel injection efficiency scenario, $\phi_{\mathbf{B}}=38^{\circ} \pm 4^{\circ}$ and $\left.\phi_{\nabla|\mathbf{B}|}=15^{\circ} \pm 15^{\circ}\right)$, smoothed with a sigma of $2^{\prime}$. Central panel: radio map of SN 1006 of Fig. 19 but smoothed with the same sigma used for the model image. Right panel: same as the left panel, but for the best-fit quasi-perpendicular scenario $\left(\phi_{\mathbf{B}} \sim 38^{\circ}\right)$.

The latter geometry would cause a centrally brightened morphology instead of two limbs, as already discussed by Orlando et al. (2007). The best-fit values of the aspect angle we derive from Fig. 20 and a conservative estimate of their uncertainties are $\phi_{\mathbf{B}}=38^{\circ} \pm 4^{\circ}$ and $\phi_{\nabla|\mathbf{B}|}=15^{\circ} \pm 15^{\circ}$.

The synthesized radio map of the best fit model is shown in Fig. 22, along with the observed radio map. Both maps have been smoothed to hide the small scale features and to focus the comparison on large spatial scales. In fact, we don't expect a pixel to pixel match between model and observations, given the simple assumptions behind the model. Indeed, the large structures of the observed radio emission are very well recovered by the best-fit model and the two images look similar.

The comparison with other gradients, namely the GRAD2, GRAD3 and GRAD4 models, shows that it is not possible to find a satisfactory fit for all the parameters. In particular, for higher values of the gradient, the angle $\theta_{D}$ is underestimated, so GRAD1 is the only model which gives us an overall good fit.

\subsubsection{Quasi-perpendicular scenario}

We also produced model images in the quasi-perpendicular injection scenario, and we repeated the analysis described in the previous paragraphs. In this case, the direction of the $\nabla|\mathbf{B}|$ is aligned with the direction of $\mathbf{B}$, so the angle $\phi_{\nabla|\mathbf{B}|}$ is the same as $\phi_{\mathbf{B}}$, and we did not consider it any further. Another consequence of this is that $R_{\max }$ is always 1 , so this parameter cannot give any diagnostic. The results are shown in Fig. 23, and it seems that a good fit can be found for $\phi_{\mathbf{B}} \sim 70^{\circ}$ (a value in agreement with the azimuthal profile analysis of Petruk et al. (2009)), even if the model points in the $\theta_{D}-C$ diagram seems to be more distant from the observed points than any scatter plot in Fig. 20, thus indicating a better fit in the quasi-parallel case than the quasi-perpendicular case.

\subsection{Discussion}

The proposed method of comparison between models of synchrotron radio emission and the real observational data of bilateral SNRs is based on the calculation of 4 morphological parameters, and we have seen that we get a good fit for a quasi-parallel injection efficiency scenario if we include a gradient of $\mathbf{B}$ and a worse fit in case of quasi-perpendicular scenario with the same gradient. This means that an agreement is found between the overall observed morphology of SN 1006 and our model, which corresponds to well defined values of the viewing geometry angles $\left(\phi_{\mathbf{B}}=38^{\circ} \pm 4^{\circ}\right.$ and $\left.\phi_{\nabla|\mathbf{B}|}=15^{\circ} \pm 15^{\circ}\right)$. We know that the angle between the SN 1006 axis of symmetry and the Galactic plane is roughly $90^{\circ}$, and that its distance is $2.2 \mathrm{kpc}$ and its Galactic latitude is $14.6^{\circ}$. Combining all this information, we can plot the direction of the magnetic field and its gradient in a 3D representation of the Galactic disk. This is shown in Fig. 24,

Remarkably, the direction of $\nabla|\mathbf{B}|$ points down toward the plane and the direction of $\mathbf{B}$ is aligned with the direction of the spiral arm near the remnant. This is indeed a very reasonable configuration for the magnetic field at this position in the Galaxy, because models of Galactic B indicates that at this location above the plane the field still retaines spiral arms features, even 

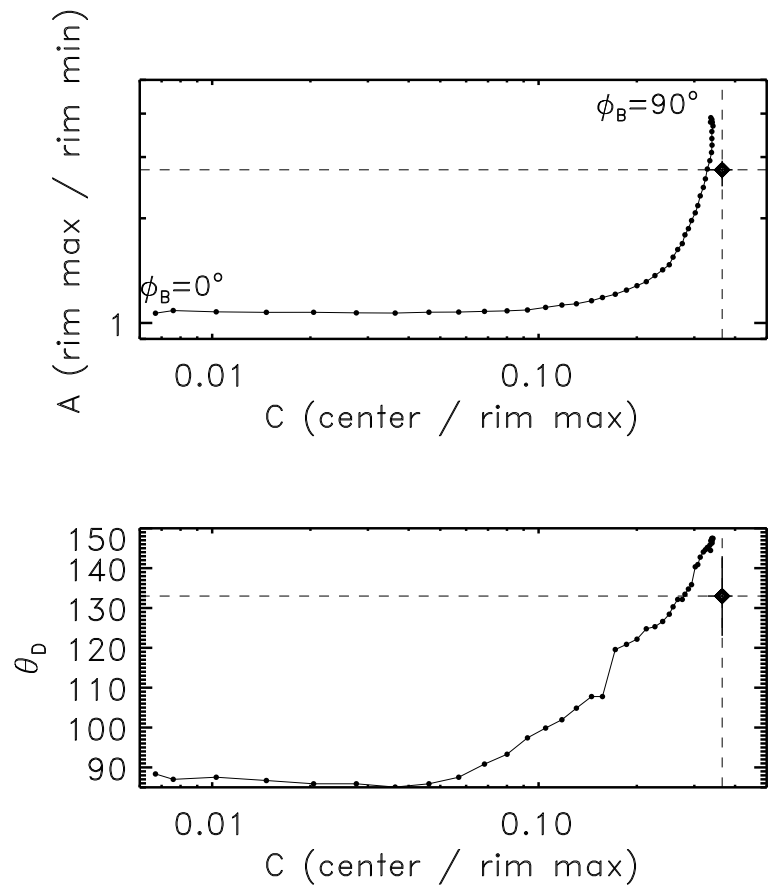

Fig. 23 Top panel: Same as Fig. 20 (top panel) but for quasiperpendicular scenario. There is no $\phi_{\nabla|\mathbf{B}|}$ angle in this case, because $\mathbf{B}$ is always aligned with $\nabla|\mathbf{B}|$. Lower panel: Scatter plot of $\theta_{D}-C$ parameters. We overplotted the values observed in SN 1006

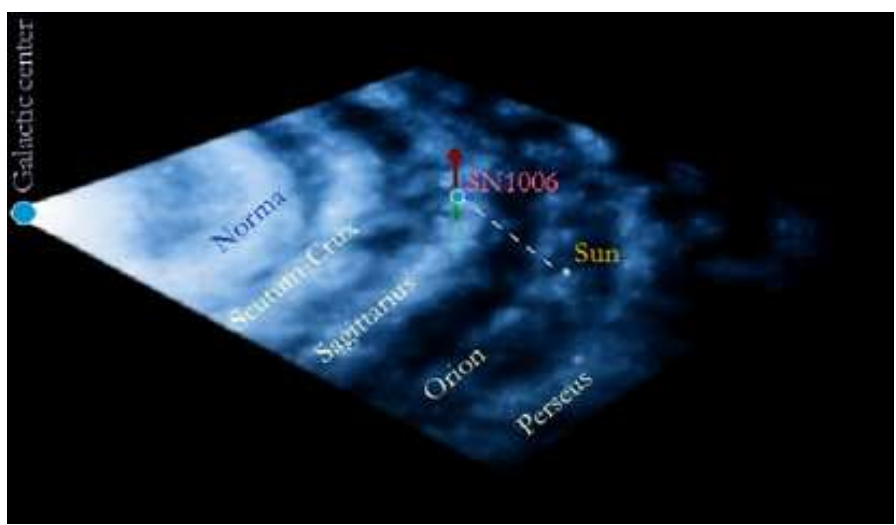

Fig. 24 Artist's impression of the magnetic field at the location of SN 1006 in our Galaxy. The red and green arrows mark the directions of the field and its gradient as derived by the best-fit model for the synchrotron radio emission of SN 1006, assuming a quasi-parallel scenario, derived in this work. The figure is to scale.

if having smaller amplitudes. Therefore, it is tempting to conclude that we are sampling the large scale field of the Galaxy. However, we note that the best-fit value of $\nabla|\mathbf{B}|$ (i.e. a variation of 1.4 times over $10 \mathrm{pc}$ ) seems to be high to be associated with the large scale $\mathbf{B}$ of the Galaxy. We have used the model of Sun et al. (2008) to compute the expected variation of the azimuthal field in the Galaxy at the location of SN 1006. In particular, we have used their $A S S+R I N G$ model which seems to be favored by rotation measures of pulsars in the plane. At a distance of $\sim 550 \mathrm{pc}$ from the plane for the SN 1006 remnant, the large-scale field is expected to vary by a factor of 1.4 on scales of more than 100 pc, much longer than the scale-length required by our model fit. This opens up the possibility that we are actually sampling the random magnetic field component. Minter \& Spangler (1996) reports length scales for this component of the order of few pc, which is compatible with the variations we derive.

\subsection{Summary and conclusion}

The synchrotron radio emission of the archetypical bilateral supernova remnant SN 1006 is compared against an MHD and emission model for this remnant, including a gradient of the external magnetic field, particle acceleration and its obliquity dependence, namely quasi-perpendicular, quasi-parallel, and isotropic scenarios for the injection efficiency. In order to explore the parameter space, which is very large due to the necessity of including the viewing geometry in the modeldata comparison, we developed a simplified procedure based on the computation of four morphological parameters. We have found a very good fit with a model assuming quasi-parallel injection efficiency and an aspect angle $\phi_{\mathbf{B}}=38^{\circ} \pm 4^{\circ}$ between the direction of $\mathbf{B}$ the line of sight, and $\phi_{\nabla|\mathbf{B}|}=15^{\circ} \pm 15^{\circ}$ between the plane of the sky and the direction of the gradient of the magnetic field, and a variation of $\mathbf{B}$ of about 1.4 times over a scale of 10 pc. A worse fit is obtained with quasi-perpendicular scenario. The overall morphology of the observed radio emission at $1.4 \mathrm{GHz}$ is correctly recovered by our best-fit model. The projected direction of $\mathbf{B}$ and $\nabla|\mathbf{B}|$ in the Galaxy are along the spiral arm and toward the plane respectively, which is in very good agreement with the expected direction of the large scale Galactic B. However, the implied gradient is too high to be associated with the large-scale Galactic $\mathbf{B}$ and more typical of the random magnetic field components.

The application of our method to selected samples of bilateral supernova remnants may yield independent estimates of the geometry of the Galactic field at several locations, which can be useful to understand the field topology in our Galaxy.

Acknowledgements SPR acknowledges support from NASA and NSF for supernova-remnant research. FB thanks his collaborators S. Orlando, O. Petruk and M. Miceli for their work on the modeling of the radio morphology of SN 1006. BMG acknowledges the support of a Federation Fellowship from the Australian Research Council. 


\section{References}

Abdo, A.A., et al. 2010a, ApJ, 710, L92

Abdo, A.A., et al. 2010b, ApJ, 714, 927

Acero, F., Ballet, J., \& Decourchelle, A. 2007, A\&A , 475, 883

Acero, F., et al. (HESS collaboration). 2009, 31st ICRC, arXiv:0907.0642

Acero, F., et al. (HESS collaboration). 2010, A\&A, 516, A62

Aharonian, F., et al. (HESS collaboration). 2005, A\&A, 435, L17

Aharonian, F., et al. (HESS collaboration). 2008, A\&A, 486, 829

Aharonian, F., et al. (HESS collaboration). 2009, ApJ, 692, 1500

Allen, G.A., Gotthelf, E.V., \& Petre, R. 1999, Proc. 26th ICRC, 3,480

Amano, T. \& Hoshino, M. 2007, ApJ , 661, 190

Atoyan, A. M. 1999, A\&A, 346, L49

Bamba, A., et al. 2003, 589, 827

Becker, R.H., et al. 1980, ApJ, 240, L33

Begelman, M. C. 1998, ApJ, 493, 291

Bell, A.R. 2004, MNRAS, 353, 550

Bell, A.R., \& Lucek, S.G. 2001, MNRAS, 321,433

Blandford, R.D., \& Eichler, D. 1987, Phys.Rep., 154, 1

Blondin, J. M., Chevalier, R. A., \& Frierson, D. M. 2001, ApJ, 563,806

Bogovalov, S. V. \& Khangoulyan, D. V. 2002, Astron. Lett., 28, 373

Borkowski, K.J., et al. 2010, ApJ, 724, L161

Bucciantini, N. 2002, A\&A, 387, 1066

-. 2010, arXiv:1005.4781

Bucciantini, N., Amato, E., \& Del Zanna, L. 2005, A\&A, 434, 189

Burn, D.J. 1966, MNRAS, 133, 67

Bykov, A.M., Uvarov, Y.A., \& Ellison, D.C. 2008, ApJ, 689, L133

Bykov, A.M., et al. 2009, MNRAS, 399, 1119

Camus, N. F., Komissarov, S. S., Bucciantini, N., \& Hughes, P. A. 2009, MNRAS, 400, 1241

Cassam-Chenaï, G., et al. 2007, ApJ, 665, 315

Chatterjee, S. \& Cordes, J, M. 2002, ApJ, 575, 407

Chevalier, R. A. 1977, in Supernovae, ed. D. N. Schramm (Dordrecht: Reidel), 53-61

Chevalier, R.A. 1982, ApJ, 258, 790

- 1998, Mem. della Soc. Ast. It., 69, 977

-. 2005, ApJ, 619, 839

Cowsik, R., \& Sarkar, S. 1980, MNRAS, 191, 855

De Jager, O.C., \& Djannati-Ataï, A. arXiv:0803.0116 1

De Luca, A., Mignani, R. P., Caraveo, P. A., \& Bignami, G. F. 2007, ApJ, 667, L77

Del Zanna, L., Amato, E., \& Bucciantini, N. 2004, A\&A, 421, 1063

Del Zanna, L., Volpi, D., Amato, E., \& Bucciantini, N. 2006, A\&A, 453, 621

DeLaney, T., Gaensler, B. M., Arons, J., \& Pivovaroff, M. J. 2006, ApJ, 640, 929

Dickel, J.R., van Breugel, W.J.M., \& Strom, R.G. 1991, AJ, 101, 2151

Dodson, R., et al. 2003, ApJ, 596, 1137

Duncan, A.R., et al. 1996, MNRAS, 280, 252

Eichler, D. 1979, ApJ, 229, 419

Ellison, D.C., \& Reynolds, S.P. 1991, ApJ, 382, 242

Ellison, D.C., et al. 2010, ApJ, 712, 287

Fan, Z., Liu, S., \& Fryer, C.L. 2010, MNRAS, 406, 1337

Favata, F., et al. 1997, A\&A, 324, L49

Fleishman, G. D. \& Bietenholz, M. F. 2007, MNRAS, 376, 625

Frail, D. A., Kassim, N. E., Cornwell, T. J., \& Goss, W. M. 1995, ApJ, 454, L129

Fulbright, M. S. \& Reynolds, S. P. 1990, ApJ , 357, 591

Gaensler, B. M. 1998, ApJ , 493, 781
Gaensler, B. M. et al. 2002, ApJ, 569, 878

2004, ApJ, 616, 383

Gelfand, J. D., Slane, P. O., \& Zhang, W. 2009, 703, 2051

Green, D.A. 2009, Bull.Astron. Soc.India, 37, 45

Hester, J. J. 2008, Ann. Rev. Astr. Ap., 46, 127

Hester, J. J., et al. 2002, ApJ, 577, L49

Hester, J.J., et al. 1995, ApJ, 448, 240

Jokipii, J.R. 1987, ApJ, 313, 842

Jun, B.-I., \& Norman, M.L. 1996, ApJ, 472, 245

Jun, B.-I., \& Jones, T.W. 1999, ApJ, 511, 774

Kalemci, E., et al. 2006, ApJ, 644, 274

Katsuda, S., et al. 2010, ApJ, 723, 383

Kennel, C. F. \& Coroniti, F. V. 1984, ApJ, 283, 694

Kesteven, M. J. \& Caswell, J. L. 1987, A\&A , 183, 118

Komissarov, S. S. \& Lyubarsky, Y. E. 2003, MNRAS, 344, L93

-. 2004, MNRAS, 349, 779

Kothes, R., Reich, W., \& Uyanıker, B. 2006, ApJ, 638, 225

Koyama, K., et al. 1995, Nature, 378, 255

Krivonos, R., et al. 2007, A\&A, 475, 775

Landecker, T.L., et al. 1999, ApJ, 527, 866

Lazendić, J.S., et al. 2004, ApJ, 602, 271

Long, K.S., et al. 2003, ApJ, 586, 1162

Lyubarsky, Y. 2002, MNRAS, 329, L34

Lyutikov, M. \& Thompson, C. 2005, ApJ, 634, 1223

Maeda, Y., et al. 2009, PASJ, 61, 1217

Matheson, H., \& Safi-Harb, S. 2005, AdvSpRes, 35, 1099

McLaughlin, M. A., et al. 2004, ApJ, 616, L131

Miceli, M., et al. 2009, A\&A , 501, 239

Minter, A. H. \& Spangler, S. R. 1996, ApJ , 458, 194

Ng, C.-Y., Gaensler, B. M., Chatterjee, S., \& Johnston, S. 2010, ApJ, 712, 596

Orlando, S., et al. 2007, A\&A , 470, 927

Ostriker, J.P., \& McKee, C.F. 1988, RevModPhys., 60, 1

Pacholczyk, A.G. 1970, Radio Astrophysics (San Francisco: Freeman)

Parizot, E., et al. 2006, A\&A, 453, 387

Patnaude, D.J., \& Fesen, R.A. 2007, AJ, 133, 147

Pavlov, G. G., Kargaltsev, O. Y., Sanwal, D., \& Garmire, G. P. 2001, ApJ, 554, L189

Petruk, O., et al. 2009, MNRAS , 393, 1034

Pohl, M., Yan, H., \& Lazarian, A. 2005, ApJ, 626, L101

Renaud, M., et al. 2006, ApJ, 647, L41

Reich, W. 2002, in Proceedings of the 270. Heraeus Seminar on Neutron Stars, Pulsars and Supernova Remnants, ed. H. L. W. Becker \& J. Trümper (Garching: MPE), 1-12

Reynolds, S.P. 1998, ApJ, 493, 375

Reynolds, S.P. 2008, ARAA, 46, 89

Reynolds, S. P. \& Chevalier, R. A. 1984, ApJ, 278, 630

Reynolds, S.P., et al. 2008, ApJ, 680, L41

Reynolds, S.P., et al. 2009, ApJ, 695, L149

Reynolds, S.P., \& Chevalier, R.A. 1981, ApJ, 245, 912

Reynolds, S.P., \& Ellison, D.C. 1992, ApJ, 399, L75

Reynolds, S.P., \& Gilmore, D.A. 1993, AJ, 106, 272

Reynoso, E., et al. 1997, ApJ, 491, 816

2004, A\&A , 425, 121

Seward, F. D., Gorenstein, P., \& Smith, R. K. 2006, ApJ, 636, 873

Slane, P.O., et al. 1999, ApJ, 525, 357

Slane, P.O., et al. 2001, ApJ, 548, 814

Slane, P., Helfand, D. J., van der Swaluw, E., \& Murray, S. S. 2004, ApJ, 616, 403

Sun, X. H., Reich, W., Waelkens, A., \& Enßlin, T. A. 2008, A\&A , 477, 573

Tamagawa, T., et al. 2009, PASJ, 61, S167

Tanaka, T., et al. 2008, ApJ, 685, 988

Tanaka, S. J. \& Takahara, F. 2010, ApJ, 715, 1248 
Toor, A. 1980, A\&A, 85, 184

Truelove, J.K., \& McKee, C.F. 1999, ApJS, 120, 299

Tziamtzis, A., Schirmer, M., Lundqvist, P., \& Sollerman, J. 2009, A\&A, 497, 167

Uchiyama, Y. 2007, Nature, 449, 576

van der Swaluw, E., Achterberg, A., \& Gallant, Y. A. 1998, Mem. della Soc. Ast. It., 69, 1017

van der Swaluw, E., Achterberg, A., Gallant, Y. A., \& Tóth, G. 2001, A\&A, 380, 309

van der Swaluw, E., Downes, T. P., \& Keegan, R. 2004, A\&A, 420, 937

Vigelius, M., et al. 2007, MNRAS, 374, 793

Vink, J., \& Laming, J.M. 2003, ApJ, 584, 758

Völk, H. J., Berezhko, E. G., \& Ksenofontov, L. T. 2003, A\&A , 409, 563

Weisskopf, M. C., et al. 2000, ApJ, 536, L81

Wilson, A. S. \& Weiler, K. W. 1976, A\&A, 53, 89

Winkler, P.F., et al. 2003, ApJ, 585, 324

Yamazaki, R., et al. 2004, A\&A , 416, 595

Yusef-Zadeh, F. \& Gaensler, B. M. 2005, Adv. Space Res., 35, 1129

Zirakashvili, V.N., \& Aharonian, F.A. 2007, A\&A, 465, 695

Zirakashvili, V.N., \& Aharonian, F.A. 2010, ApJ, 708, 965 